\title{
Validity and Sensitivity of an Inertial Measurement Unit-Driven Biomechanical Model of Motor Variability for Gait
}

\author{
Christopher A. Bailey $^{1}\left(\mathbb{D}\right.$, Thomas K. Uchida ${ }^{2}$, Julie Nantel ${ }^{1}(\mathbb{D})$ and Ryan B. Graham ${ }^{1, *(D)}$ \\ 1 School of Human Kinetics, University of Ottawa, Ottawa, ON K1N 6N5, Canada; \\ cbailey2@uottawa.ca (C.A.B.); jnantel@uottawa.ca (J.N.) \\ 2 Department of Mechanical Engineering, University of Ottawa, Ottawa, ON K1N 6N5, Canada; \\ thomas.uchida@uottawa.ca \\ * Correspondence: ryan.graham@uottawa.ca
}

Citation: Bailey, C.A.; Uchida, T.K.; Nantel, J.; Graham, R.B. Validity and Sensitivity of an Inertial

Measurement Unit-Driven Biomechanical Model of Motor Variability for Gait. Sensors 2021, 21, 7690. https://doi.org/10.3390/ s21227690

Academic Editor: James F. Rusling

Received: 27 September 2021

Accepted: 16 November 2021

Published: 19 November 2021

Publisher's Note: MDPI stays neutral with regard to jurisdictional claims in published maps and institutional affiliations.

Copyright: (c) 2021 by the authors. Licensee MDPI, Basel, Switzerland. This article is an open access article distributed under the terms and conditions of the Creative Commons Attribution (CC BY) license (https:/ / creativecommons.org/licenses/by/ $4.0 /)$.

\begin{abstract}
Motor variability in gait is frequently linked to fall risk, yet field-based biomechanical joint evaluations are scarce. We evaluated the validity and sensitivity of an inertial measurement unit (IMU)-driven biomechanical model of joint angle variability for gait. Fourteen healthy young adults completed seven-minute trials of treadmill gait at several speeds and arm swing amplitudes. Trunk, pelvis, and lower-limb joint kinematics were estimated by IMU- and optoelectronic-based models using OpenSim. We calculated range of motion (ROM), magnitude of variability (meanSD), local dynamic stability $\left(\lambda_{\max }\right)$, persistence of ROM fluctuations (DFA $\alpha$ ), and regularity (SaEn) of each angle over 200 continuous strides, and evaluated model accuracy (RMSD: root mean square difference), consistency ( $\mathrm{ICC}_{2,1}$ : intraclass correlation), biases, limits of agreement, and sensitivity to within-participant gait responses (effects of speed and swing). RMSDs of joint angles were 1.7-9.2 (pooled mean of $4.8^{\circ}$ ), excluding ankle inversion. ICCs were mostly good to excellent in the primary plane of motion for ROM and in all planes for meanSD and $\lambda_{\max }$, but were poor to moderate for DFA $\alpha$ and SaEn. Modelled speed and swing responses for ROM, meanSD, and $\lambda_{\text {max }}$ were similar. Results suggest that the IMU-driven model is valid and sensitive for field-based assessments of joint angle time series, ROM in the primary plane of motion, magnitude of variability, and local dynamic stability.
\end{abstract}

Keywords: gait; inertial measurement unit; joint kinematics; local dynamic stability; OpenSim; persistence; regularity; stride-to-stride variability

\section{Introduction}

Possessing too much or too little motor variability, the natural variability in sensorimotor actions [1], is well linked to walking-related fall risk. These actions include whole-body and joint motions that vary over time from stride to stride. Elderly fallers exhibit greater stride-to-stride variability in spatiotemporal outputs (e.g., stride time) compared to non-fallers [2,3]. This difference may emerge from altered stride-to-stride joint angle patterns that have been observed with older age, including lower local dynamic stability (measured by the local divergence exponent) [4], lower regularity (measured by the sample entropy) [5], and a shift in the magnitude of variability in ankle motion from the sagittal to the frontal plane (measured by the standard deviation) [6]. For example, calculation of phase-dependent entropy of trunk motion during walking was recently shown to improve prediction of future single-time fallers in community-dwelling older adults [7]. Monitoring the variability of joint angles in aging adults at a larger scale could help better understand the salient elements of stride-to-stride control that predict falls, which could better individualize fall-prevention interventions. However, measurements of joint angles (and variability from stride to stride) typically rely on optoelectronic motion capture systems that are expensive, require training and expertise to operate, and involve intensive data acquisition and processing procedures. Optoelectronic motion capture of 
gait is also restricted to treadmills [4,5] or short distances overground [6], which may not fully replicate the stride-to-stride variability that occurs over longer distances and durations overground. Using this motion capture approach, large-scale evaluations of motor variability in realistic and clinically-relevant gait scenarios are infeasible.

Wearable inertial measurement units (IMUs) offer an alternative technology for estimating joint angles that can address these limitations of optoelectronic motion capture. IMUs have been used to estimate joint kinematics since 1990 [8], with recent work showing that IMU-based kinematic models of lower-limb activities achieve absolute differences (i.e., accuracies) ranging from 1 to $10^{\circ}$ relative to optoelectronic-based models [9-15] and good-excellent consistencies in the sagittal plane time series $[9,12,16,17]$. Some IMU-based kinematic models are built based on machine learning approaches [13], but most others typically involve (i) estimating the IMU orientation by fusing accelerometer and gyroscope data, (ii) estimating the anatomical segment orientation by applying a sensor-to-segment calibration (e.g., [18]), and (iii) calculating joint angles as the relative orientation between reference frames fixed to adjacent body segments (see [19] for review). A particular obstacle to the longer-duration recordings necessary to measure stride-to-stride kinematics is preventing IMU sensor orientation drift attributed to sensor fusion. Strap-down integration of an IMU on a non-stabilized segment amplifies random noise in linear accelerations and angular velocities, leading to drift in the estimated orientations. Many sensor fusion algorithms address horizontal drift by incorporating data on the Earth's magnetic field detected by the magnetometer [20-24]; however, detection of the magnetic field is disturbed locally by ferromagnetic materials [25]. Beyond the magnetometer, drift corrections are possible over short durations by adding zero-velocity updates [26] or over short distances by adding localization using technologies like ultrawideband [11], but these approaches do not provide a solution for long durations and distances.

Anatomical joint constraints in the underlying kinematic model can help to mitigate drift over long durations and distances. Using the OpenSense toolkit to compute inverse kinematics of a biomechanical model with IMU inputs, Al Borno et al. [15] recently demonstrated root mean squared differences (RMSD) of 3-6 for IMU-based hip flexion, hip abduction, knee flexion, and ankle dorsiflexion angles relative to optoelectronic motion capture, with near-zero drift (from 0.14 to $0.17^{\circ} / \mathrm{min}$ ) over ten minutes of walking. This supports the earlier findings of Kok et al. [27] and provides a new opensource option for constructing an IMU-based kinematic model. This inverse kinematics approach to solving joint angles has the added benefit of mitigating experimental (i.e., non-biological) noise [28], which could particularly benefit evaluations of motor variability. Although constrained optimization problems like inverse kinematics can require high computation time to solve, recent work from Slade et al. [14] demonstrated that a real-time IMU-based solution is possible. They reported RMSD in joint angles of approximately $5^{\circ}$, a difference accepted as reasonable for many clinical applications [29]. The OpenSense extension of OpenSim [30,31] provides the first open-source platform for IMU-based biomechanical modelling and a free alternative to cost-prohibitive and closed-source commercial models [20]. Because Al Borno et al. [15] used the magnetometer to calculate drift-free kinematics and Slade et al. [14] reported kinematic drifts in their magnetometer-free solution, it remains unclear whether a magnetometer-free, IMU-based biomechanical model can provide accurate joint angles during gait beyond one minute duration. Furthermore, it is unknown whether IMU-based biomechanical models are valid for evaluating stride-tostride variability or are sufficiently sensitive to changes in gait kinematics for evaluating fall risk.

The goal of this study was to validate a magnetometer-free, open-source, IMU-based biomechanical model of joint angles and stride-to-stride variability for a moderate duration of continuous gait. We determined the concurrent validity of IMU-based and optoelectronicbased model measurements of joint angles and joint angle variability from the trunk down using OpenSim, and determined the sensitivity of discrete measurements of joint angles and joint angle variability to different gait conditions. We hypothesized that joint angle 
and joint angle variability measurements from the IMU-based model would be accurate and consistent with the optoelectronic-based model, and that changes in angles and angle variability under different gait conditions would be detected similarly in the two models.

\section{Methods}

\subsection{Participants}

Fourteen healthy young adults ( 7 males, 7 females) were recruited as a convenience sample from the Ottawa, Canada area. While the IMU-based model is intended for use in older adults as well, young adults were tested to first establish feasibility of the methods and modelling. This sample size ( $n=12$, with 2 extra to account for possible data attrition) was determined a priori using $G^{*}$ Power [32] based on our sensitivity analyses and reflects the number of participants needed to detect a large effect size (partial $\eta^{2} \geq 0.14$ ) for a within-group factor with three measurement levels (speed: preferred, slow, fast; swing: preferred, active, bound) at a power of 0.80 and an $\alpha$ of 0.05 . Participants were excluded if they had a musculoskeletal injury in the preceding 6 months, or any chronic neurological or orthopaedic disorders. Participants all provided written informed consent to this study, which followed the Declaration of Helsinki and was approved by the University of Ottawa Research Ethics Board (H-01-21-6261).

\subsection{Instrumentation}

Following informed consent, the participants donned spandex motion capture pants and their own athletic shoes. Participants were then instrumented for optoelectronicand IMU-based motion capture (Figure 1). An 11-camera optoelectronic system (Vantage, Vicon, Oxford, UK) sampled trajectories of spherical retroreflective markers at $120 \mathrm{~Hz}$ using Nexus 2.11 (Vicon, Oxford, UK). Markers were placed on the participant's body using double-sided tape, following the marker locations used with the full-body model for gait simulations in OpenSim from Rajagopal et al. (Link: https://simtk.org/projects/full_body (accessed on 1 March 2021)) [33]. Anatomical markers were placed on each wrist (radial and ulnar styloid process), on each elbow (medial and lateral epicondyle), on the trunk (left and right acromion process, right clavicular head, spinous process of C7), on the pelvis (left and right anterior superior iliac spine, left and right posterior superior iliac spine), on each knee (medial and lateral femoral condyle), on each ankle (medial and lateral malleolus), and on each foot (heel, 1st and 5th metatarsal head). The dynamic marker set was modified to add redundancy such that rigid-body clusters of four markers (rather than three) were positioned using Velcro straps on the trunk and each forearm, arm, thigh, and shank. Anatomical markers on the feet, pelvis, and the lateral malleoli doubled as dynamic markers for these segments.

IMU-based motion capture was performed using a platform of eight wearable sensors (Dot, Xsens, Enschede, The Netherlands) and a mobile application (Xsens Dot Precision Motion Tracking) for synchronized acquisition of the raw accelerometer and gyroscope data from each sensor. IMU sensors were positioned on the feet (top of the shoe), shanks (anterior aspect, distal and immediately above the malleoli), thighs (anterior aspect, around the largest circumference), pelvis (posterior aspect under the posterior superior iliac spines), and trunk (posterior aspect at the level of the sternum). IMUs were oriented such that the positive $x y z$ axes in the sensor frame in anatomical position were directed leftward, upward, and forward, respectively. Raw accelerations and angular velocities were sampled at $60 \mathrm{~Hz}$ using the mobile application in data logging mode.

\subsection{Experimental Procedure}

Following instrumentation, preferred gait speed was identified according to the procedure of Dingwell and Marin [34]. With the participant blinded to the speed and walking slowly on the treadmill, gait speed was progressively increased until they reported that the speed was "faster than preferred". Speed was then progressively decreased until they 
reported that the speed was "slower than preferred". This sequence was then repeated three times, with the average of the six speeds defined as the preferred gait speed.

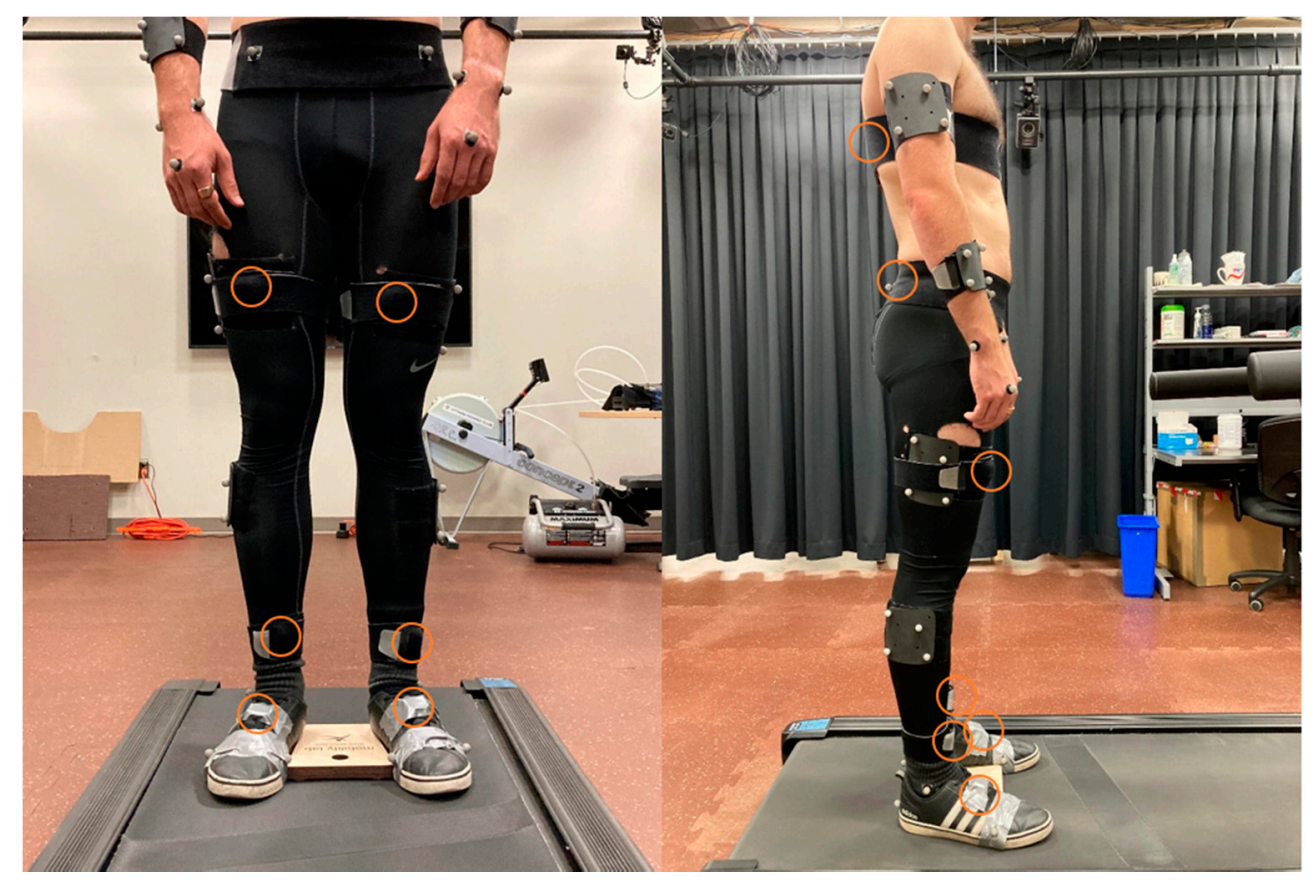

Figure 1. Participant in standing pose on the treadmill showing the dynamic marker set, inertial measurement unit positions (strapped to segments, circled in orange), and the wooden block.

Following a static calibration of the anatomical markers with the participant in standing pose, the participant completed five gait trials on the treadmill (Horizon Fitness, Cottage Grove, WI, USA). Each trial began with a 30 s procedure to warm up the sensors, with the feet oriented on the treadmill using a wooden block to minimize inter-trial and inter-individual differences in foot excursion posture (Figure 1). The participant stood quietly for the first $5 \mathrm{~s}$, leaned forward for the next $10 \mathrm{~s}$, then returned to quiet standing for the final $15 \mathrm{~s}$. After this baseline procedure, the block was removed and the participant completed seven minutes of walking. This sequence was repeated for five trials, each under a different gait condition that varied by gait speed and/or arm swing magnitude: (1) preferred gait speed and arm swing; (2) 70\% preferred gait speed, preferred arm swing; (3) $130 \%$ preferred gait speed, preferred arm swing; (4) preferred gait speed, active arm swing (the participant was instructed to swing their arms such that forward swing peaked when the arm was horizontal); and (5) preferred gait speed, arms bound to the torso (using straps across the arms and across the elbows). These gait conditions with different speeds and arm swing amplitudes were evaluated since they have been shown to alter stride-tostride variability patterns in gait [35-39], providing a basis for exploring the sensitivity of the IMU-based model. Gait conditions each lasted seven minutes to record at least six minutes of consecutive and constant-speed strides. This duration was needed to reach a minimum of 150 consecutive strides for stable measurements of motor variability [40] and is also the duration of the six-minute walk test [41], a common assessment of functional mobility. Condition order was randomized, the participant rested for a minimum of three minutes between trials, and optoelectronic and IMU data were continuously sampled during each trial.

\subsection{Data Analysis}

\subsubsection{Optoelectronic-Based Biomechanical Modelling}

Marker trajectories were labelled, gap-filled with a Woltring spline [42], and lowpass filtered at $10 \mathrm{~Hz}$ using Nexus (v2.11, Vicon Inc., Oxford, UK). Filtered trajectories were then imported into OpenSim v4.1 [31] and used to simulate motion of a full-body 
model containing 37 degrees of freedom (DOF) and 80 muscle-tendon units actuating the lower limbs [33]. This model includes a 3-DOF trunk (relative to pelvis; flexion, lateral bending, rotation), a 6-DOF pelvis (relative to ground; tilt, obliquity, rotation), 3-DOF hips (flexion, abduction, rotation), 1-DOF knees (flexion), 2-DOF ankles (flexion, inversion), and 1-DOF metatarsophalangeal joints (flexion). For simplicity and consistency, angles in the sagittal, frontal, and transverse planes will be described as flexion/extension (FE), abduction/adduction (AA), and internal/external rotation (IE), respectively. The 1-DOF toe joints were locked since toe motion was not recorded by IMUs. The model was scaled to the participant using the positions of the anatomical markers in the static trial; joint angles in each trial were then computed via an inverse kinematic analysis that minimized the least-squared distance between each pair of experimental and model markers. Upperlimb markers were not included in the analysis since no IMUs were placed on the upper limbs and applying weights to the upper-limb markers that equaled weights of lowerlimb markers did not influence the inverse kinematics solution (Supplementary Material: Figure S1). Marker weights were manually selected to minimize the root-mean-square error over all marker pairs, resulting in equal weights except for weights of twice the magnitude for markers on the acromion processes (trunk), anterior and posterior superior iliac spines (pelvis), and lateral malleoli (shanks).

\subsubsection{IMU-Based Biomechanical Modelling}

Using Matlab (R2020b, The MathWorks Inc., Natick, MA, USA), raw linear accelerations and angular velocities were fused offline to calculate sensor orientations using the magnetometer-free algorithm of Madgwick et al. [21]. Orientation drifts were then removed using a detrending procedure. Beginning at the $20 \mathrm{~s}$ timestamp $(5 \mathrm{~s}$ after the participant had completed the forward lean and was standing quietly) and endingj at trial completion, quaternions were converted to ZYX Euler angles and fit to a function using 'polyfit'. Linear drift was identified as slope less than $-0.0010 \mathrm{rad} / \mathrm{s}$ or greater than $0.0010 \mathrm{rad} / \mathrm{s}$ and removed from the Euler angle signal using 'detrend', then Euler angles were converted back to quaternion representation. An example showing the orientation of a sensor before and after drift removal is provided in the Supplementary Material (Figure S2).

Detrended sensor quaternions were imported into OpenSim using the OpenSense toolkit to simulate motion of the same full-body biomechanical model [33]. Sensor-tosegment registration was performed to associate the orientation of each sensor with the corresponding segment in the model; specifically, each thigh, shank, and foot sensor was registered, respectively, to each femur, tibia, and talus body segment. Sensor orientations were converted from their local coordinate systems to the OpenSim coordinate system using the following sequence of body-fixed rotations: $180^{\circ}$ about $x$, then $90^{\circ}$ about $y$, and finally $-90^{\circ}$ about $\mathrm{z}$. IMU segment frames were identified based on the standing pose at the start of each gait trial: fixed rotational offsets were applied to recorded IMU sensor frames based on the segment frames of the biomechanical model in a neutral standing pose (i.e., joint flexion of $0^{\circ}$ ), with heading offsets applied to individual IMU sensor frames to match the average heading and align with the anterior-posterior axis of the biomechanical model [14,15]. As with the optoelectronic-based model, joint angles in each trial were calculated via inverse kinematics; for the IMU-based model, the solver minimized axis-angle differences between the IMU segment orientations and IMU sensor orientations [15]. We compensated for differences between the initial pose of the optoelectronic and IMU models by offsetting optoelectronic-based joint angle time series by a constant to match the neutral standing pose of the IMU model. In contrast with the single ankle dorsiflexion/plantarflexion DOF modelled previously [14,15], we chose to also model ankle inversion/eversion since both sagittal- and frontal-plane ankle compensations are relevant to gait of aging adults [6]. We explored optoelectronic- and IMU-based inverse kinematic solutions of the 1-DOF and 2-DOF ankle models and confirmed that the additional ankle DOF did not affect the inverse kinematics solutions for other lower-limb joint angles (Supplementary Material: Figure S1). 
Example animations of the optoelectronic- and IMU-based biomechanical models can be viewed in the Supplementary Material (Videos S1-S4).

\subsection{Calculation of Kinematic Outcomes}

Time series of optoelectronic- and IMU-modelled joint angles and linear velocities of the foot segments were exported to Matlab. Strides were partitioned by the first and subsequent heel strike events, identified from the tri-dimensional linear velocity vectors of the calcaneus segments. Linear velocities were low-pass Butterworth filtered with zero lag $(10 \mathrm{~Hz}$ cutoff, fourth-order) and the Euclidean norm linear velocity vector was then calculated. From the Euclidean norm linear velocity, heel strike events were identified as the local minima immediately following each local maximum (Supplementary Material: Figure S3). After removing strides in the first $30 \mathrm{~s}$ to ensure gait was at steady state, the raw and time-normalized series (101 points per stride: 0-100\%) of joint angles were analyzed for the subsequent 200 strides. (This was the minimum number of synchronized strides available for all trials and participants.) Five outcomes were then calculated for each joint angle, as described below.

\subsubsection{Range of Motion (ROM)}

The difference between the maximum and minimum angles for each stride in the time-normalized series. The mean value across strides was computed.

\subsubsection{Mean Standard Deviation (meanSD)}

A measure of the absolute magnitude of variability, meanSD was calculated using the time-normalized series. SD was calculated across all strides for each time point ( $n=101$ points) and the mean SD across time points (meanSD) was computed.

\subsubsection{Maximum Finite-Time Lyapunov Exponent $\left(\lambda_{\max }\right)$}

A measure of local dynamic stability, $\lambda_{\max }$ (i.e., the local divergence exponent) was calculated using the continuous series. The continuous series was normalized to 20,000 points (100 per stride on average), then $\lambda_{\max }$ was computed with 5 embedding dimensions at a lag of 10 points from $0-0.5$ strides (50 points) $[4,43]$. $\lambda_{\max }$ measures the local divergence of neighbouring trajectories with higher positive values indicative of higher divergence and lower local dynamic stability.

\subsubsection{Detrended Fluctuation Analysis Scaling Exponent (DFA $\alpha$ )}

A measure of statistical persistence, DFA $\alpha$ was calculated as the fluctuation in ROM across strides, computed as previously described [44,45] and quantifying the extent to which ROM fluctuations statistically persist. DFA $\alpha$ is non-negative and unitless, with values greater than 0.5 indicating persistence (i.e., a fluctuation is typically followed by a fluctuation in the same direction), values less than 0.5 indicating anti-persistence (i.e., a fluctuation is typically followed by a fluctuation in the opposite direction), and values around 0.5 indicating no correlation between consecutive fluctuations [46].

\subsubsection{Sample Entropy (SaEn)}

A measure of signal regularity, SaEn was calculated using the continuous series, 2 embedding dimensions, and a 0.15 tolerance distance [47]. SaEn can be investigated at several scales using a multiscale function; we selected a scale factor of 4 which is believed to be the approximate value where entropy of physiological signals stabilizes during selfselected slow, normal (usual), and fast walking speeds [47]. To compensate for the influence of sampling frequency [48], IMU-based joint angles were resampled at $120 \mathrm{~Hz}$ to match the optoelectronic system. SaEn is non-negative and unitless, with higher values indicating lower regularity. 


\subsection{Statistical Analyses}

\subsubsection{Analyses of IMU-Model Validity}

Statistical analyses were completed using SPSS (v27, IBM, Armonk, NY, USA). For each degree of freedom, we assessed concurrent validity of the IMU-based joint angles and angle outputs relative to the optoelectronic-based joint angles and angle outputs for 1000 strides (5 gait conditions $\times 200$ strides). Mean RMSD was calculated for the time-normalized joint angle series as well as for each outcome variable. For the time series analysis, relative difference was calculated as the coefficient of variation of RMSD relative to the optoelectronic-based ROM ( $\left.\mathrm{CV}_{\text {rom }}\right)$; for the outcome variable analysis, relative difference was calculated relative to the optoelectronic-based mean $\left(\mathrm{CV}_{\text {mean }}\right)$. Intraclass correlation coefficients ( $\left.\mathrm{ICC}_{2,1}\right)$ and Bland-Altman plot metrics (IMU-optoelectronic measurement bias, 95\% limits of agreement) were computed to examine consistency and agreement between outcomes. ICC $\mathrm{IC}_{2,1}$ values less than 0.40 , from 0.40 to 0.59 , from 0.60 to 0.74 , and greater than or equal to 0.75 were interpreted as poor, fair, good, and excellent consistency, respectively [49]. Biases indicated whether IMU-based outcomes were overestimated (positive bias) or underestimated (negative bias) on average.

\subsubsection{Analyses of IMU-Model Sensitivity}

For each degree of freedom and outcome, we assessed the sensitivity of the IMU model to detect the same within-participant changes as the marker-based model by conducting repeated measures ANOVAs on each model to test for effects of gait speed (speed: preferred, $70 \%$ preferred, 130\% preferred) and arm swing (swing: preferred, active, bound). Greenhouse-Geisser corrections were applied if sphericity was violated, and critical alpha was set to 0.027 using the Benjamini-Hochberg procedure to account for false discovery rate due to multiple comparisons [50] (240 $p$-values: 2 models $\times 2$ statistical effects $\times 12$ angles $\times 5$ outcomes). Post hoc tests, comparing each condition to preferred speed and preferred arm swing gait, were made with Bonferroni corrections $(p<0.05)$.

\section{Results}

\subsection{Participant Characteristics}

Participant height, mass, and BMI (mean \pm SD) averaged $1.72 \pm 0.07 \mathrm{~m}, 69.6 \pm 14.2 \mathrm{~kg}$, and $23.5 \pm 3.9 \mathrm{~kg} / \mathrm{m}^{2}$, respectively. Gait at preferred speed averaged $1.12 \pm 0.18 \mathrm{~m} / \mathrm{s}$ (range: $0.72-1.50$ ), at $70 \%$ preferred speed averaged $0.79 \pm 0.13 \mathrm{~m} / \mathrm{s}$ (range: $0.50-1.05 \mathrm{~m} / \mathrm{s}$ ), and at $130 \%$ preferred speed averaged $1.46 \pm 0.24 \mathrm{~m} / \mathrm{s}$ (range: $0.93-1.95$ ).

\subsection{IMU-Model Validity}

\subsubsection{Validity of Joint Angle Time Series}

Mean values are presented in Table 1, with ensemble averaged curves for the preferred speed condition displayed in Figure 2. Based on values pooled across conditions, mean RMSD was less than $5^{\circ}$ for all trunk angles, pelvis AA and IE, hip FE, and knee FE, with all other angles except pelvis FE and ankle AA approaching the $5^{\circ}$ threshold. RMSD pooled across conditions and angles was $5.3^{\circ}$, which dropped to $4.8^{\circ}$ when ankle AA was excluded. RMSDs were consistent across 200 consecutive strides, showing that IMU-modelled joint angles did not drift (Supplementary Material: Figure S4). $\mathrm{CV}_{\text {rom }}$ averaged $26.9 \%$ across angles, being lowest in the transverse plane for the trunk $(16.2 \%)$ and pelvis $(12.8 \%)$, and lowest in the sagittal plane for the hip $(9.2 \%)$, knee $(6.4 \%)$, and ankle $(17.1 \%)$.

\subsubsection{Validity of Joint Angle Range of Motion and Motor Variability Outcomes}

Mean values are presented in Table 2, with Bland-Altman plots for each outcome displayed in Supplementary Material (Figures S5-S9). Good-excellent consistency was seen for ROM of trunk IE, pelvis AA, pelvis IE, hip FE, and ankle FE ( $\left.\mathrm{ICC}_{2,1}: 0.62-0.85\right)$, for meanSD of all angles except knee FE and ankle AA (ICC $\left.{ }_{2,1}: 0.60-0.80\right)$, for $\lambda_{\max }$ of all angles except trunk IE and ankle AA (ICC $2,1: 0.67-0.89$ ), for DFA $\alpha$ of trunk IE and ankle 
FE (ICC $2,1: 0.62-0.65)$, and for SaEn of trunk AA, pelvis AA, pelvis IE, and knee FE (ICC 2,1 : $0.61-0.74)$.

Table 1. Root mean squared differences (RMSD) and RMSD relative to optoelectronic-modelled range of motion ( $\left.C V_{\text {rom }}\right)$ of the IMU-modelled joint angle time series during gait $(\mathrm{N}=200$ strides). Values are group means [95\% confidence intervals]. Highlights represent pooled differences accepted as reasonable (green: RMSD $\leq 5.0^{\circ}$ ), differences approaching reasonable levels (yellow: $5.0^{\circ}<$ RMSD $\leq 7.0^{\circ}$ ), and differences exceeding reasonable levels (orange: $\operatorname{RMSD}>7.0^{\circ}$ ).

\begin{tabular}{|c|c|c|c|c|c|c|c|}
\hline & \multirow[t]{2}{*}{ Angle } & \multicolumn{6}{|l|}{ Gait Condition } \\
\hline & & $\begin{array}{l}\text { Preferred Speed, } \\
\text { Preferred Swing }\end{array}$ & $\begin{array}{l}70 \% \text { Preferred } \\
\text { Speed, } \\
\text { Preferred } \\
\text { Swing }\end{array}$ & $\begin{array}{l}\text { 130\% Preferred } \\
\text { Speed, } \\
\text { Preferred } \\
\text { Swing }\end{array}$ & $\begin{array}{l}\text { Preferred } \\
\text { Speed, Active } \\
\text { Swing }\end{array}$ & $\begin{array}{l}\text { Preferred Speed, } \\
\text { Arms Bound }\end{array}$ & Pooled \\
\hline \multirow{14}{*}{ RMSD $\left({ }^{\circ}\right)$} & Trunk FE & $3.8[2.1,5.5]$ & $4.1[2.1,5.5]$ & $4.2[2.2,6.2]$ & $4.2[2.8,5.6]$ & $3.4[2.0,4.8]$ & $4.0[2.3,5.6]$ \\
\hline & Trunk AA & $3.6[2.8,4.3]$ & $3.6[2.7,4.5]$ & $3.9[3.1,4.7]$ & $4.3[3.3,5.2]$ & $3.4[2.8,4.0]$ & $3.7[2.9,4.5]$ \\
\hline & Trunk IE & $4.0[3.1,5.0]$ & $2.9[2.5,3.3]$ & $3.8[3.2,4.4]$ & $6.7[1.9,11.4]$ & $2.9[2.5,3.4]$ & $4.1[2.6,5.5]$ \\
\hline & Pelvis FE & $10.6[7.7,13.5]$ & $9.6[6.7,12.5]$ & $8.1[5.2,10.9]$ & $9.2[6.1,12.2]$ & $8.8[5.4,12.2]$ & $9.2[6.2,12.3]$ \\
\hline & Pelvis AA & $2.1[1.7,2.5]$ & $2.3[1.7,2.8]$ & $2.2[1.9,2.5]$ & $2.4[1.9,2.5]$ & $2.0[1.6,2.4]$ & $2.2[1.8-2.6]$ \\
\hline & Pelvis IE & $1.9[1.5,2.2]$ & $1.7[1.3,2.2]$ & $2.1[1.7,2.5]$ & $2.0[1.6,2.4]$ & $1.8[1.5,2.2]$ & $1.9[1.5,2.3]$ \\
\hline & Hip FE & $4.4[3.5,5.2]$ & $3.7[3.0,4.4]$ & $5.8[4.9,6.8]$ & $4.2[3.4,4.9]$ & $4.5[3.0,6.0]$ & $4.5[3.6,5.5]$ \\
\hline & Hip AA & $5.5[4.6,6.4]$ & $4.8[4.0,5.6]$ & $5.8[4.9,6.7]$ & $5.4[4.4,6.4]$ & $5.5[4.6,6.5]$ & $5.4[4.5,6.3]$ \\
\hline & Hip IE & $6.1[5.4,6.8]$ & $5.8[4.9,6.6]$ & $6.5[5.7,7.2]$ & $5.8[5.0,6.7]$ & $6.7[5.5,7.9]$ & $6.2[5.3,7.0]$ \\
\hline & Knee FE & $4.6[3.7,5.5]$ & $3.7[3.1,4.3]$ & $4.8[3.6,5.9]$ & $4.0[3.6,4.4]$ & $4.7[3.7,5.6]$ & $4.3[3.5,5.1]$ \\
\hline & Ankle FE & $6.7[5.6,7.9]$ & $6.2[4.9,7.5]$ & $7.5[6.2,8.8]$ & $6.9[5.4,8.4]$ & $7.4[6.0,8.8]$ & $6.9[5.6,8.3]$ \\
\hline & Ankle AA & $12.0[10.3,13.6]$ & $10.2[8.8,11.5]$ & $12.0[10.7,13.2]$ & $11.5[10.1,12.9]$ & $12.7[10.9,14.5]$ & $11.7[10.2,13.2]$ \\
\hline & Pooled (all) & $5.4[5.0,5.9]$ & $4.9[4.5,5.2]$ & $5.6[5.2,5.9]$ & $5.5[5.0,6.0]$ & $5.3[4.9,5.8]$ & $5.3[4.9,5.8]$ \\
\hline & Pooled (without Ankle AA) & $4.8[4.4,5.3]$ & $4.4[4.1,4.7]$ & $5.0[4.6,5.4]$ & $5.0[4.4,5.6]$ & $4.6[4.2,5.1]$ & $4.8[4.3,5.2]$ \\
\hline \multirow{14}{*}{$\mathrm{CV}_{\text {rom }}(\%)$} & Trunk FE & $25.5[16.6,34.3]$ & $27.9[17.8,38.1]$ & $29.4[16.9,42.0]$ & $27.7[19.6,35.8]$ & $28.2[17.5,38.9]$ & $27.7[17.7,37.8]$ \\
\hline & Trunk AA & $15.0[13.0,17.1]$ & $16.8[13.7,20.0]$ & $15.3[13.0,17.7]$ & $17.7[14.5,20.8]$ & $17.9[15.1,19.2]$ & $16.6[13.9,19.2]$ \\
\hline & Trunk IE & $15.6[14.2,37.8]$ & $14.2[12.0,16.5]$ & $13.7[11.3,16.1]$ & $22.1[3.8,40.4]$ & $15.6[13.7,17.5]$ & $16.2[10.5,21.9]$ \\
\hline & Pelvis FE & $120.3[80.3,160.3]$ & $94.2[64.7,123.7]$ & $95.3[55.5,135.0]$ & $92.9[59.5,126.2]$ & $107.3[58.5,156.0]$ & $102.0[63.7,140.2]$ \\
\hline & Pelvis AA & $12.3[10.5,14.1]$ & $15.2[11.0,19.4]$ & $12.0[10.5,13.5]$ & $13.4[11.8,15.1]$ & $14.4[11.2,17.7]$ & $13.5[11.0,16.0]$ \\
\hline & Pelvis IE & $13.4[10.4,16.3]$ & $12.9[9.2,16.5]$ & $12.8[10.7,14.9]$ & $11.7[9.9,13.6]$ & $13.2[10.8,15.7]$ & $12.8[10.2,15.4]$ \\
\hline & Hip FE & $9.1[7.5,10.6]$ & $8.5[6.6,10.5]$ & $11.0[9.3,12.6]$ & $8.0[6.6,9.4]$ & $9.2[6.7,11.8]$ & $9.2[7.3,11.0]$ \\
\hline & Hip AA & $23.3[18.5,28.2]$ & $21.9[17.2,26.5]$ & $22.9[18.8,27.0]$ & $22.1[17.3,27.0]$ & $28.2[21.5,35.0]$ & $23.7[18.7,28.7]$ \\
\hline & Hip IE & $32.2[27.5,37.0]$ & $30.2[25.0,36.1]$ & $32.9[29.6,36.1]$ & $29.8[25.4,34.1]$ & $35.0[29.2,40.9]$ & $32.0[27.3,36.7]$ \\
\hline & Knee FE & $6.7[5.4,8.0]$ & $5.5[4.6,6.4]$ & $7.0[5.5,8.5]$ & $6.0[5.4,6.7]$ & $6.8[5.3,8.3]$ & $6.4[5.2,7.6]$ \\
\hline & Ankle FE & $16.2[13.9,18.5]$ & $17.2[14.3,20.2]$ & $17.7[15.2,20.2]$ & $16.6[13.3,19.9]$ & $17.6[14.5,20.6]$ & $17.1[14.3,19.9]$ \\
\hline & Ankle AA & $46.1[39.6,52.5]$ & $41.4[35.4,47.4]$ & $46.1[40.8,51.3]$ & $43.5[37.3,49.6]$ & $49.8[43.0,56.5]$ & $45.3[39.2,51.5]$ \\
\hline & Pooled (all) & $28.0[23.9,32.1]$ & $25.5[22.5,28.5]$ & $26.3[22.7,30.0]$ & $26.0[22.2,29.7]$ & $28.6[24.2,33.0]$ & $26.9[23.1,30.7]$ \\
\hline & Pooled (without Ankle AA) & $26.3[22.2,30.5]$ & $24.1[21.0,27.1]$ & $24.5[20.7,28.4]$ & $24.4[20.5,28.3]$ & $26.7[21.9,31.5]$ & $25.2[21.2,29.2]$ \\
\hline
\end{tabular}

FE: flexion/extension; AA: abduction/adduction; IE: internal/external rotation.

Differences [absolute: RMSD (relative: $\mathrm{CV}_{\text {mean }}$ )] between outcomes with good-excellent consistency were $2.0-7.7^{\circ}(5.0-38.3 \%)$ for ROM, $0.14-0.61^{\circ}(10.9-50.0 \%)$ for meanSD, $0.20-0.61$ (3.9-17.8\%) for $\lambda_{\max }, 0.16-0.17(16.6-17.2 \%)$ for $\mathrm{DFA} \alpha$, and $0.07-0.24(15.1-63.1 \%)$ for SaEn.

Significant mean biases (defined as cases where the $95 \%$ confidence interval did not cross zero; Table 2) indicated that the IMU model underestimated ROM of the trunk, pelvis, and ankle FE by $0.4-7.0^{\circ}$, but overestimated ROM of frontal plane hip and ankle angles

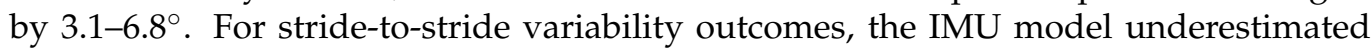
meanSD of trunk IE and pelvis AA by $0.08-0.52^{\circ}, \lambda_{\max }$ of trunk IE by $0.23, \mathrm{DFA} \alpha$ of pelvis AA and hip AA by 0.07 , and SaEn of pelvis FE, hip AA, hip IE, knee FE, and ankle AA by $0.02-0.12$. These outcomes were more frequently overestimated by the IMU model, with higher meanSD $\left(0.04-0.56^{\circ}\right)$ and $\lambda_{\max }(0.20-1.13)$ for most angles, higher DFA $\alpha$ of ankle AA (0.12), and higher SaEn of trunk AA, trunk IE, pelvis AA, pelvis IE, hip FE, and ankle FE (0.04-0.19). These biases approximated the optoelectronic-measured interindividual standard deviations in our sample and in measurements from other studies for joint angle ROM [51], meanSD [35,39], DFA $\alpha$ [39], and SaEn [5,39], but exceeded inter-individual standard deviations for joint angle $\lambda_{\max }[4,39]$. The Bland-Altman plots (Supplementary Material: Figures S5-S9) show that nearly all measurement differences (14 participants $\times 5$ conditions $=70$ values) fell within the $95 \%$ limits of agreement, with 
few outliers in ROM $(\mathrm{N}=1-5)$, meanSD $(\mathrm{N}=2-5), \lambda_{\max }(\mathrm{N}=1-4), \mathrm{DFA} \alpha(\mathrm{N}=1-5)$, and SaEn $(\mathrm{N}=0-6)$ of individual angles.

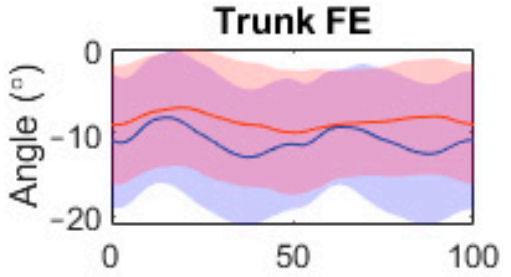

Pelvis FE

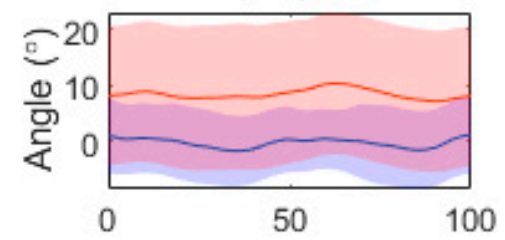

Hip FE

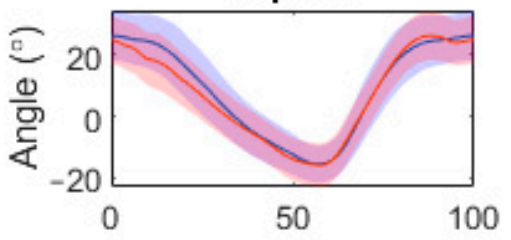

Knee FE

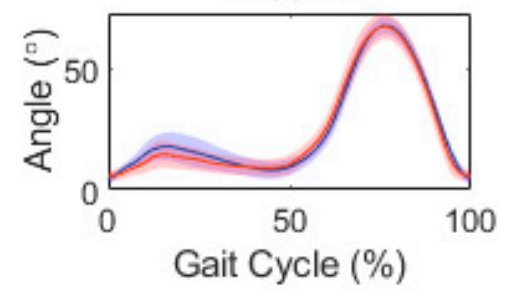

Trunk AA

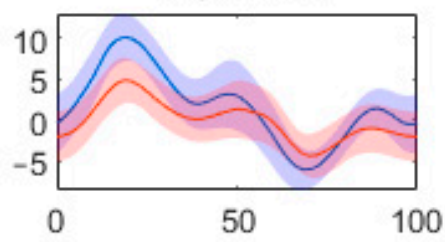

Pelvis AA

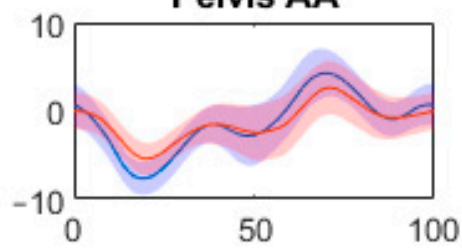

Hip AA

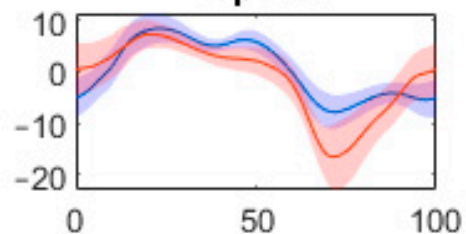

Ankle FE

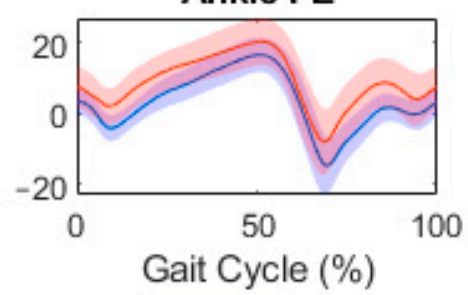

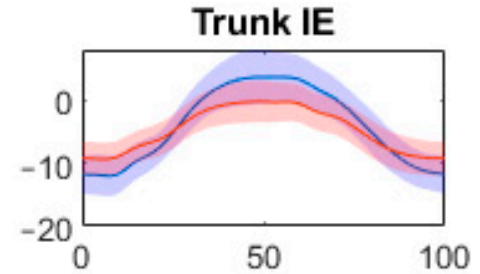

Pelvis IE

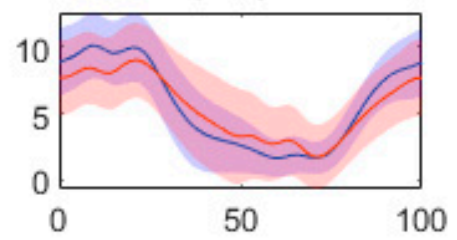

Hip IE

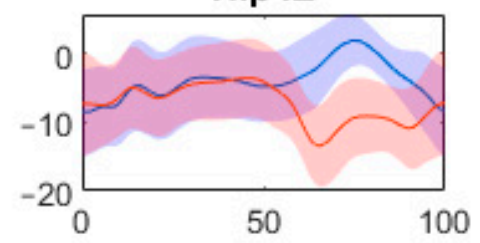

Ankle AA

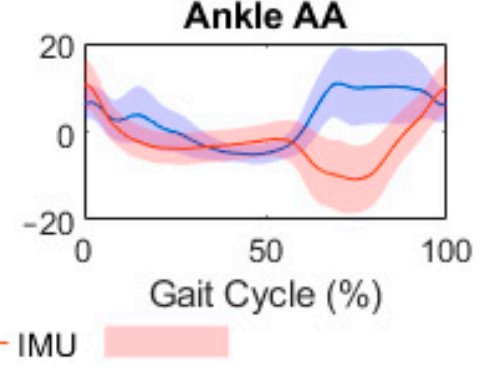

Figure 2. Ensemble averaged joint angles from the optoelectronic and inertial measurement unit (IMU) models during gait at preferred speed and with preferred arm swing. Angles include flexion/extension (FE), abduction/adduction (AA), and internal/external rotation (IE).

\subsection{IMU-Model Sensitivity}

Precise descriptives (means, 95\% confidence intervals) and statistical effects ( $p$-values) for each outcome can be found in Supplementary Material (videos S1 and S2). Since consistency was generally poor to moderate for DFA $\alpha$ and SaEn, indicating a lack of acceptable concurrent validity, the sensitivity of these outcomes was not explored.

ROM (Figure 3). Speed effects: The models detected similar responses relative to preferred-speed gait, with decreased ROM of trunk AA, trunk IE, pelvis AA, hip FE, hip IE, and ankle FE at 70\% preferred speed, and increased ROM of trunk AA, trunk IE, pelvis AA, pelvis IE, hip angles, and ankle FE at $130 \%$ preferred speed. Differing responses, where only one model detected a significant change, were found at $70 \%$ preferred speed for pelvis IE, hip AA, knee FE, and ankle AA, and at 130\% preferred speed for ankle AA. The changes detected, however, followed the same trends of decreased ROM at $70 \%$ preferred speed and increased ROM at $130 \%$ preferred speed. Swing effects: The models also detected similar changes relative to preferred arm swing during active swing, with increased ROM of pelvis IE and hip FE, decreased ROM of knee FE, and no change in ROM of trunk FE, trunk AA, pelvis FE, pelvis AA, hip AA, hip IE, ankle FE, and ankle AA. With arms bound, both models detected decreased ROM of trunk IE and pelvis AA, and no change for pelvis FE, hip FE, hip IE, ankle FE, and ankle AA. 
Table 2. Validity of IMU-modelled vs. optoelectronic-modelled joint angle outcomes for gait. Outcomes are range of motion (ROM), mean standard deviation (meanSD), local divergence exponent $\left(\lambda_{\max }\right)$, detrended fluctuation analysis scaling exponent for range of motion (DFA $\alpha)$, and sample entropy (SaEn). Validity metrics are intraclass correlation coefficients ( $\mathrm{ICC}_{2,1}$ ), root mean square difference (RMSD), coefficient of variation of the optoelectronic mean (CV mean), IMUoptoelectronic bias, and 95\% limits of agreement (LOA $95 \%$ ). Values are group means [ $95 \%$ confidence intervals]. Highlights represent excellent (dark green: $\mathrm{ICC}_{2,1} \geq 0.75$ ), good (green: $0.60 \leq \mathrm{ICC}_{2,1}<0.75$ ), fair (yellow: $0.40 \leq \mathrm{ICC}_{2,1}<0.60$ ), and poor (orange: $\mathrm{ICC}_{2,1}<0.40$ ) consistency.

\begin{tabular}{|c|c|c|c|c|c|c|c|}
\hline & \multirow{2}{*}{ Angle } & \multirow{2}{*}{$\mathrm{ICC}_{2,1}$} & \multirow{2}{*}{ RMSD } & \multirow{2}{*}{$\mathrm{CV}_{\text {mean }}$} & \multirow{2}{*}{ Bias } & \multicolumn{2}{|l|}{ LOA $_{95 \%}$} \\
\hline & & & & & & Lower & Upper \\
\hline \multirow{12}{*}{ ROM } & Trunk FE & $0.13[-0.11,0.35]$ & $2.5[2.1,2.9]$ & $26.1[22.3,29.8]$ & $-1.3[-1.8,-0.8]^{*}$ & $-5.4[-5.9,-4.9]$ & $2.8[2.3,3.3]$ \\
\hline & Trunk AA & $0.48[0.28,0.64]$ & $7.8[7.0,8.6]$ & $41.3[38.3,44.4]$ & $-7.0[-7.8,-6.2]^{*}$ & $-13.6[-14.3,-12.8]$ & $-0.5[-1.3,0.3]$ \\
\hline & Trunk IE & $0.85[0.77,0.90]$ & $7.7[6.8,8.6]$ & $38.3[34.8,41.7]$ & $-6.7[-7.6,-5.8]^{*}$ & $-14.1[-15.0,-13.2]$ & $0.7[-0.2,1.6]$ \\
\hline & Pelvis FE & $0.03[-0.26,0.21]$ & $1.8[1.5,2.1]$ & $26.2[20.8,31.5]$ & $-0.4[-0.8,0.0]^{*}$ & $-3.8[-4.2,-3.4]$ & $3.0[2.6,3.4]$ \\
\hline & Pelvis AA & $0.62[0.46,0.75]$ & $4.8[4.2,5.3]$ & $32.6[28.5,35.6]$ & $-4.1[-4.7,-3.6]^{*}$ & $-8.8[-9.4,-8.2]$ & $0.5[0.0,1.1]$ \\
\hline & Pelvis IE & $0.84[0.75,0.90]$ & $2.0[1.7,2.4]$ & $14.8[12.1,17.6]$ & $-0.5[-0.9,0.0]^{*}$ & $-4.4[-4.9,-3.9]$ & $3.5[3.0,4.0]$ \\
\hline & Hip FE & $0.84[0.75,0.90]$ & $2.8[2.4,3.3]$ & $5.0[3.8,6.1]$ & $0.1[-0.6,0.8]$ & $-5.5[-6.2,-4.8]$ & $5.7[5.0,6.4]$ \\
\hline & Hip AA & $0.24[-0.01,0.45]$ & $8.5[7.5,9.6]$ & $44.8[36.9,52.7]$ & $6.8[5.5,8.0]^{*}$ & $-3.5[-4.9,-2.3]$ & $17.1[15.8,18.3]$ \\
\hline & Hip IE & $-0.11[-0.35,-0.13]$ & $4.8[4.2,5.4]$ & $36.9[30.4,43.4]$ & $0.4[-0.7,1.6]$ & $-9.1[-10.2,-7.9]$ & $9.9[8.7,11.0]$ \\
\hline & Knee FE & $0.26[0.02,0.47]$ & $6.9[5.9,7.9]$ & $8.8[7.2,10.3]$ & $-1.4[-3.0,0.2]$ & $-14.7[-16.4,13.1]$ & $11.9[10.3,13.5]$ \\
\hline & Ankle FE & $0.76[0.64,0.85]$ & $4.7[4.1,5.4]$ & $12.4[10.3,14.5]$ & $-1.2[-2.3,-0.1]^{*}$ & $-10.3[-11.4,-9.2]$ & $7.8[6.7,8.9]$ \\
\hline & Ankle AA & $0.10[-0.14,0.34]$ & $8.4[7.2,9.6]$ & $33.0[26.6,39.3]$ & $3.1[1.2,5.0]^{*}$ & $-12.3[-14.2,-10.4]$ & $18.5[16.6,20.3]$ \\
\hline \multirow{12}{*}{ meanSD } & Trunk FE & $0.63[0.46,0.75]$ & $0.29[0.25,0.34]$ & $17.8[14.1,21.6]$ & $0.07[0.00,0.14]^{*}$ & $-0.49[-0.56,-0.42]$ & $0.63[0.56,0.70]$ \\
\hline & Trunk AA & $0.79[0.68,0.86]$ & $0.18[0.15,0.22]$ & $10.9[8.6,13.1]$ & $0.02[-0.02,0.07]$ & $-0.34[-0.38,-0.29]$ & $0.38[0.34,0.43]$ \\
\hline & Trunk IE & $0.80[0.70,0.87]$ & $0.60[0.54,0.66]$ & $36.3[33.6,39.1]$ & $-0.52[-0.59,-0.45]^{*}$ & $-1.10[-1.17,-1.03]$ & $0.06[-0.01,0.13]$ \\
\hline & Pelvis FE & $0.77[0.65,0.85]$ & $0.16[0.13,0.18]$ & $12.7[10.1,15.2]$ & $0.04[0.00,0.07]^{*}$ & $-0.26[-0.30,-0.22]$ & $0.33[0.30,0.37]$ \\
\hline & Pelvis AA & $0.60[0.43,0.73]$ & $0.14[0.12,0.17]$ & $12.7[10.2,15.2]$ & $-0.08[-0.11,-0.05]^{*}$ & $-0.32[-0.35,-0.29]$ & $0.16[0.13,0.19]$ \\
\hline & Pelvis IE & $0.64[0.48,0.76]$ & $0.28[0.23,0.32]$ & $19.7[15.1,24.2]$ & $0.20[0.15,0.24]^{*}$ & $-0.19[-0.242,-0.15]$ & $0.59[0.54,0.63]$ \\
\hline & Hip FE & $0.80[0.69,0.87]$ & $0.23[0.20,0.26]$ & $17.5[14.4,20.6]$ & $0.16[0.12,0.20]^{*}$ & $-0.16[-0.20,-0.12]$ & $0.48[0.44,0.52]$ \\
\hline & Hip AA & $0.70[0.56,0.81]$ & $0.14[0.12,0.16]$ & $12.6[10.1,15.0]$ & $0.06[0.03,0.09]^{*}$ & $-0.18[-0.21,-0.15]$ & $0.30[0.27,0.33]$ \\
\hline & Hip IE & $0.69[0.54,0.80]$ & $0.20[0.17,0.23]$ & $12.5[9.9,15.1]$ & $0.10[0.06,0.14]^{*}$ & $-0.24[-0.28,-0.20]$ & $0.44[0.40,0.48]$ \\
\hline & Knee FE & $0.48[0.27,0.65]$ & $0.40[0.34,0.46]$ & $20.7[15.6,25.9]$ & $0.19[0.10,0.27]^{*}$ & $-0.51[-0.60,-0.43]$ & $0.89[0.80,0.97]$ \\
\hline & Ankle FE & $0.68[0.53,0.79]$ & $0.61[0.55,0.66]$ & $50.0[43.5,56.5]$ & $0.56[0.50,0.62]^{*}$ & $0.10[-0.04,0.16]$ & $1.02[0.97,1.08]$ \\
\hline & Ankle AA & $0.24[0.00,0.46]$ & $0.38[0.32,0.43]$ & $25.6[20.7,30.5]$ & $0.20[0.13,0.28]^{*}$ & $-0.42[-0.49,-0.34]$ & $0.83[0.75,0.90]$ \\
\hline \multirow{12}{*}{$\lambda_{\max }$} & Trunk FE & $0.72[0.58,0.82]$ & $0.54[0.48,0.60]$ & $14.3[12.3,16.2]$ & $0.44[0.37,0.52]^{*}$ & $-0.18[-0.25,-0.10]$ & $1.06[0.99,1.14]$ \\
\hline & Trunk AA & $0.85[0.77,0.91]$ & $0.40[0.36,0.44]$ & $10.5[9.3,11.7]$ & $0.36[0.32,0.40]^{*}$ & $0.04[-0.00,0.08]$ & $0.69[0.65,0.72]$ \\
\hline & Trunk IE & $0.59[0.40,0.73]$ & $0.34[0.29,0.39]$ & $6.5[5.3,7.7]$ & $-0.23[-0.29,-0.17]^{*}$ & $-0.73[-0.79,-0.67]$ & $0.27[0.21,0.33]$ \\
\hline & Pelvis FE & $0.89[0.82,0.93]$ & $0.61[0.56,0.66]$ & $17.8[16.1,19.5]$ & $0.58[0.52,0.63]^{*}$ & $0.16[0.10,0.21]$ & $1.00[0.95,1.05]$ \\
\hline & Pelvis AA & $0.82[0.71,0.88]$ & $0.37[0.32,0.41]$ & $10.1[8.6,11.6]$ & $0.32[0.27,0.36]^{*}$ & $-0.05[-0.10,-0.01]$ & $0.69[0.64,0.73]$ \\
\hline & Pelvis IE & $0.67[0.51,0.78]$ & $0.22[0.19,0.25]$ & $3.9[3.1,4.7]$ & $-0.04[-0.09,0.01]$ & $-0.47[-0.52,-0.42]$ & $0.39[0.34,0.44]$ \\
\hline & Hip FE & $0.87[0.80,0.92]$ & $0.20[0.17,0.22]$ & $4.2[3.5,4.9]$ & $-0.04[-0.09,0.01]$ & $-0.42[-0.47,-0.37]$ & $0.34[0.29,0.39]$ \\
\hline & Hip AA & $0.78[0.66,0.86]$ & $0.31[0.28,0.34]$ & $8.4[7.5,9.4]$ & $0.27[0.23,0.30]^{*}$ & $-0.02[-0.05,0.02]$ & $0.56[0.52,0.59]$ \\
\hline & Hip IE & $0.75[0.62,0.84]$ & $0.44[0.39,0.49]$ & $12.7[10.9,14.5]$ & $0.38[0.33,0.43]^{*}$ & $-0.06[-0.11,0.00]$ & $0.82[0.77,0.87]$ \\
\hline & Knee FE & $0.74[0.61,0.84]$ & $0.40[0.34,0.46]$ & $7.4[5.9,8.9]$ & $0.20[0.12,0.28]^{*}$ & $-0.48[-0.56,-0.40]$ & $0.88[0.79,0.96]$ \\
\hline & Ankle FE & $0.74[0.60,0.83]$ & $0.45[0.41,0.50]$ & $13.0[11.4,14.5]$ & $0.40[0.35,0.45]^{*}$ & $-0.02[-0.07,0.03]$ & $0.82[0.77,0.87]$ \\
\hline & Ankle AA & $0.37[0.14,0.56]$ & $1.17[1.10,1.25]$ & $51.1[45.9,56.4]$ & $1.13[1.05,1.21]^{*}$ & $0.49[0.41,0.56]$ & $1.77[1.69,1.85]$ \\
\hline \multirow{12}{*}{ DFA $\alpha$} & Trunk FE & $0.40[0.18,0.58]$ & $0.21[0.18,0.24]$ & $22.7[17.2,28.2]$ & $-0.03[-0.08,0.02]$ & $-0.44[-0.49,-0.39]$ & $0.38[0.33,0.43]$ \\
\hline & Trunk AA & $0.27[0.04,0.48]$ & $0.19[0.16,0.22]$ & $18.7[14.8,22.6]$ & $-0.05[-0.09,0.00]$ & $-0.42[-0.46,-0.37]$ & $0.32[0.28,0.37]$ \\
\hline & Trunk IE & $0.65[0.49,0.76]$ & $0.17[0.15,0.20]$ & $16.6[13.0,20.2]$ & $-0.00[-0.04,0.04]$ & $-0.34[-0.38,-0.30]$ & $0.34[0.30,0.38]$ \\
\hline & Pelvis FE & $0.36[0.14,0.55]$ & $0.20[0.17,0.23]$ & $25.5[20.4,30.7]$ & $0.01[-0.04,0.05]$ & $-0.39[-0.44,-0.25]$ & $0.40[0.35,0.45]$ \\
\hline & Pelvis AA & $0.48[0.28,0.64]$ & $0.21[0.18,0.24]$ & $21.2[17.7,24.7]$ & $-0.07[-0.12,-0.03]^{*}$ & $-0.47[-0.52,-0.42]$ & $0.32[0.27,0.37]$ \\
\hline & Pelvis IE & $0.46[0.25,0.63]$ & $0.16[0.14,0.19]$ & $17.6[13.3,21.9]$ & $0.03[-0.01,0.06]$ & $-0.29[-0.33,-0.25]$ & $0.34[0.30,0.38]$ \\
\hline & Hip FE & $0.50[0.30,0.66]$ & $0.20[0.17,0.23]$ & $21.0[16.8,25.3]$ & $-0.02[-0.06,0.03]$ & $-0.41[-0.46,-0.36]$ & $0.38[0.33,0.43]$ \\
\hline & Hip AA & $0.37[0.14,0.56]$ & $0.23[0.20,0.27]$ & $21.3[17.4,25.1]$ & $-0.07[-0.12,-0.01]^{*}$ & $-0.50[-0.56,-0.45]$ & $0.37[0.32,0.43]$ \\
\hline & Hip IE & $0.13[-0.12,0.36]$ & $0.24[0.21,0.27]$ & $26.7[21.8,31.6]$ & $-0.04[-0.10,0.02]$ & $-0.51[-0.56,-0.45]$ & $0.43[0.37,0.48]$ \\
\hline & Knee FE & $0.43[0.21,0.61]$ & $0.20[0.17,0.23]$ & $20.0[16.1,23.9]$ & $0.02[-0.03,0.07]$ & $-0.37[-0.42,-0.32]$ & $0.41[0.36,0.46]$ \\
\hline & Ankle FE & $0.62[0.44,0.75]$ & $0.16[0.13,0.18]$ & $17.2[13.4,21.0]$ & $0.02[-0.02,0.06]$ & $-0.29[-0.33,-0.25]$ & $0.33[0.29,0.37]$ \\
\hline & Ankle AA & $-0.05[-0.29,0.20]$ & $0.31[0.35,0.26]$ & $35.9[28.2,43.7]$ & $0.12[0.05,0.19]^{*}$ & $-0.44[-0.51,-0.38]$ & $0.68[0.61,0.75]$ \\
\hline \multirow{12}{*}{ SaEn } & Trunk FE & $0.56[0.38,0.70]$ & $0.18[0.15,0.20]$ & $17.0[13.8,20.2]$ & $0.02[-0.02,0.06]$ & $-0.33[-0.37,-0.29]$ & $0.36[0.32,0.41]$ \\
\hline & Trunk AA & $0.61[0.44,0.74]$ & $0.19[0.16,0.21]$ & $29.8[23.3,36.3]$ & $0.15[0.13,0.18]^{*}$ & $-0.05[-0.08,-0.03]$ & $0.36[0.34,0.39]$ \\
\hline & Trunk IE & $0.48[0.28,0.64]$ & $0.10[0.08,0.12]$ & $16.1[12.3,19.9]$ & $0.04[0.01,0.06]^{*}$ & $-0.15[-0.17,-0.13]$ & $0.22[0.20,0.24]$ \\
\hline & Pelvis FE & $0.53[0.34,0.68]$ & $0.22[0.19,0.26]$ & $16.7[14.1,19.4]$ & $-0.12[-0.16,-0.07]^{*}$ & $-0.49[-0.54,-0.45]$ & $0.26[0.22,0.31]$ \\
\hline & Pelvis AA & $0.68[0.54,0.79]$ & $0.12[0.10,0.13]$ & $15.1[12.1,18.1]$ & $0.06[0.03,0.08]^{*}$ & $-0.15[-0.17,-0.12]$ & $0.26[0.23,0.28]$ \\
\hline & Pelvis IE & $0.64[0.48,0.76]$ & $0.24[0.21,0.27]$ & $63.1[47.9,78.4]$ & $0.19[0.15,0.22]^{*}$ & $-0.11[-0.14,-0.07]$ & $0.48[0.45,0.52]$ \\
\hline & Hip FE & $0.44[0.23,0.62]$ & $0.05[0.04,0.06]$ & $17.9[14.3,21.4]$ & $0.04[0.03,0.04]^{*}$ & $-0.04[-0.05,-0.03]$ & $0.11[0.10,0.12]$ \\
\hline & Hip AA & $0.17[-0.08,0.39]$ & $0.15[0.13,0.17]$ & $23.5[20.0,27.0]$ & $-0.09[-0.12,-0.06]^{*}$ & $-0.33[-0.36,-0.30]$ & $0.15[0.12,0.18]$ \\
\hline & Hip IE & $-0.12[-0.35,0.12]$ & $0.25[0.22,0.29]$ & $21.3[17.2,25.4]$ & $-0.04[-0.10,-0.02]^{*}$ & $-0.53[-0.59,-0.47]$ & $0.46[0.40,0.52]$ \\
\hline & Knee FE & $0.74[0.61,0.83]$ & $0.07[0.05,0.08]$ & $16.5[12.8,20.2]$ & $-0.02[-0.04,-0.01]^{*}$ & $-0.14[-0.16,-0.13]$ & $0.10[0.08,0.11]$ \\
\hline & Ankle FE & $0.46[0.24,0.63]$ & $0.13[0.11,0.14]$ & $24.6[20.2,29.0]$ & $0.10[0.08,0.12] *$ & $-0.06[-0.08,-0.04]$ & $0.26[0.24,0.28]$ \\
\hline & Ankle AA & $0.10[-0.14,0.34]$ & $0.19[0.16,0.22]$ & $26.8[22.1,31.6]$ & $-0.03[-0.08,-0.01]^{*}$ & $-0.40[-0.44,-0.35]$ & $0.34[0.29,0.38]$ \\
\hline
\end{tabular}

FE: flexion/extension; AA: abduction/adduction; IE: internal/external rotation. * significant bias (95\% confidence interval did not cross zero). 


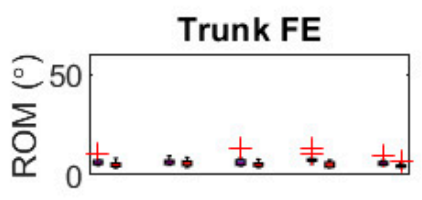

Pelvis FE

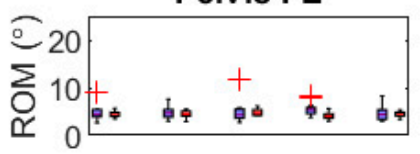

Hip FE
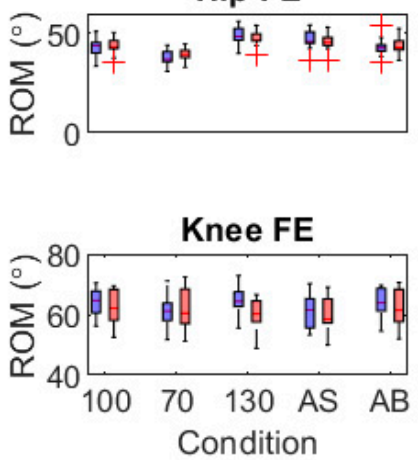

Trunk AA

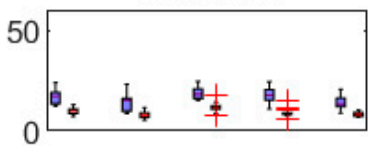

Pelvis AA

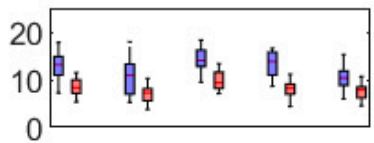

Hip AA

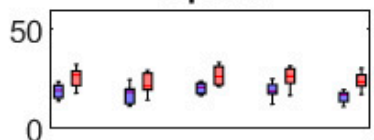

Ankle FE

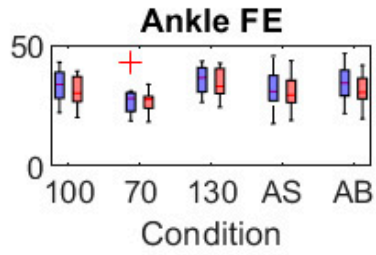

Trunk IE

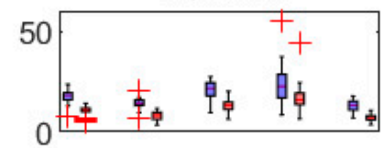

Pelvis IE

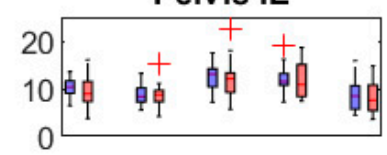

Hip IE

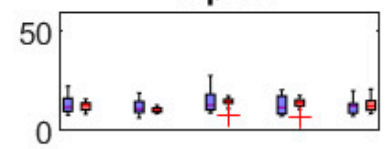

Ankle AA

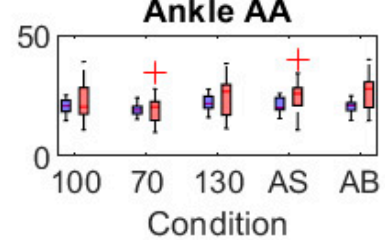

Figure 3. Boxplots of range of motion (ROM) of trunk, pelvis, and lower-limb joint angles for the optoelectronic-driven (blue) and IMU-driven (red) biomechanical models of constant-speed treadmill gait. Conditions displayed on the horizontal axis are preferred speed and arm swing (100), 70\% preferred speed (70), 130\% preferred speed (130), active arm swing (AS), and arms bound ( $\mathrm{AB})$. Red crosses are outliers $>2 *$ interquartile range. Angles include flexion/extension (FE), abduction/adduction (AA), and internal/external rotation (IE).

meanSD (Figure 4). Speed effects: The models detected similar non-responses to speed changes for meanSD of trunk FE, trunk IE, ankle FE, and ankle AA. However, optoelectronic-based model increases in meanSD at 70\% preferred speed (trunk AA, pelvis FE, pelvis AA, hip FE, hip AA, knee FE) and at 130\% preferred speed (pelvis IE) went undetected by the IMU-based model. Swing effects: Relative to preferred arm swing, the models detected similar increases in meanSD (trunk AA, pelvis AA, pelvis IE, hip AA, hip IE) and non-responses (trunk FE, hip FE, knee FE) during active arm swing, as well as similar non-responses with arms bound (trunk FE, pelvis FE, pelvis AA, and all hip, knee, and ankle angles). Optoelectronic-detected increases in meanSD of trunk AA and IE and decreases for pelvis IE with arms bound went undetected by the IMU-based model.

$\lambda_{\max }$ (Figure 5). Speed effects: The models detected similar responses to speed changes in $\lambda_{\max }$ of joint angles with few exceptions. Both models detected increases in $\lambda_{\max }$ at $70 \%$ preferred speed (trunk FE, trunk AA, pelvis-down angles), decreases in $\lambda_{\max }$ at $130 \%$ preferred speed (trunk FE, pelvis FE, pelvis AA, hip AA, hip IE, ankle AA), and no response in $\lambda_{\text {max }}$ of knee FE at $130 \%$ preferred speed. Swing effects: The models also detected similar $\lambda_{\text {max }}$ responses relative to preferred arm swing, with increases in trunk FE, pelvis angles, hip AA, hip IE, and ankle FE during active swing, and decreases in trunk IE, but increases in pelvis IE, with arms bound. Similar non-responses in $\lambda_{\max }$ of ankle AA during active swing and of trunk FE, hip FE, hip AA, knee FE, ankle FE, and ankle AA with arms bound were also seen. 

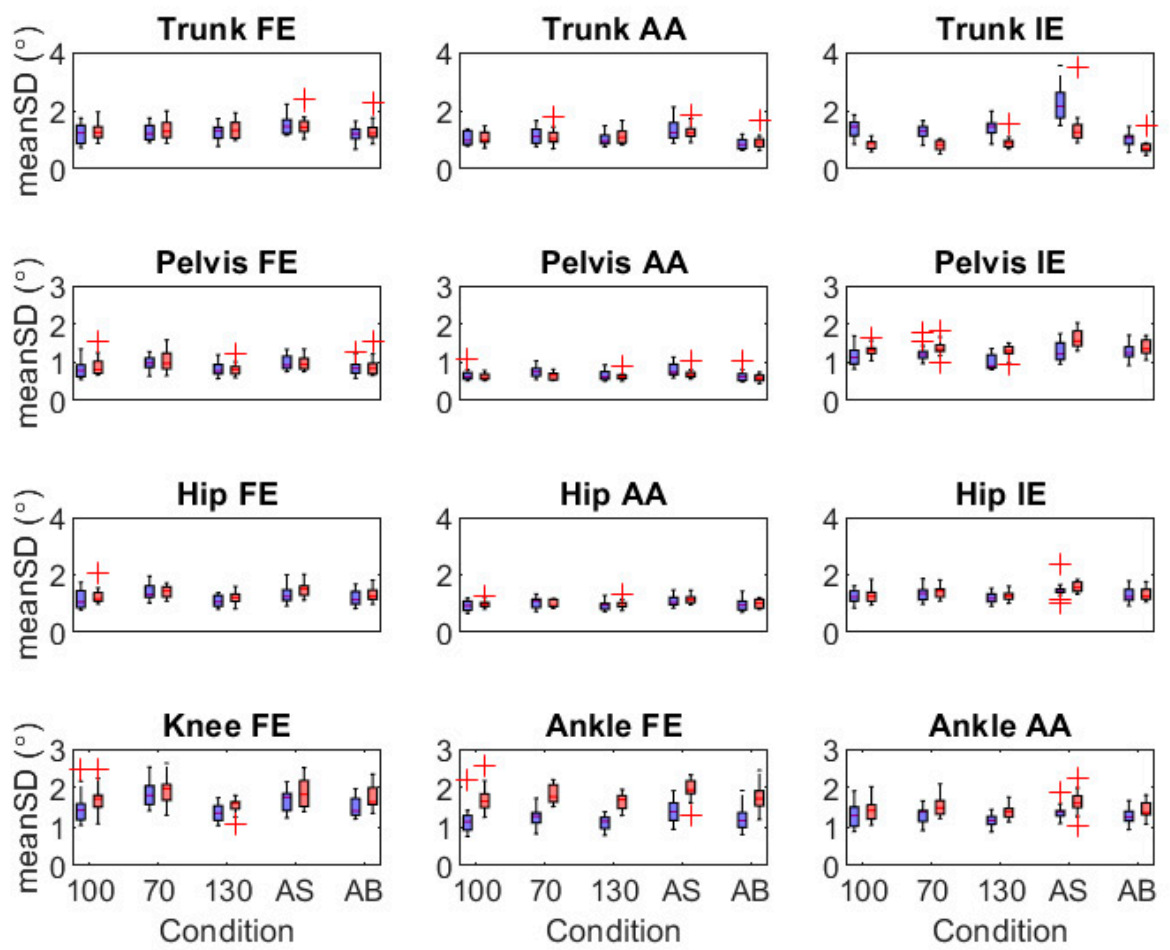

Figure 4. Boxplots of mean standard deviation (meanSD) of trunk, pelvis, and lower-limb joint angles for the optoelectronicdriven (blue) and IMU-driven (red) biomechanical models of constant-speed treadmill gait. Conditions displayed on the horizontal axis are preferred speed and arm swing (100), 70\% preferred speed (70), 130\% preferred speed (130), active arm swing (AS), and arms bound (AB). Red crosses are outliers $>2 *$ interquartile range. Angles include flexion/extension (FE), abduction/adduction (AA), and internal/external rotation (IE).
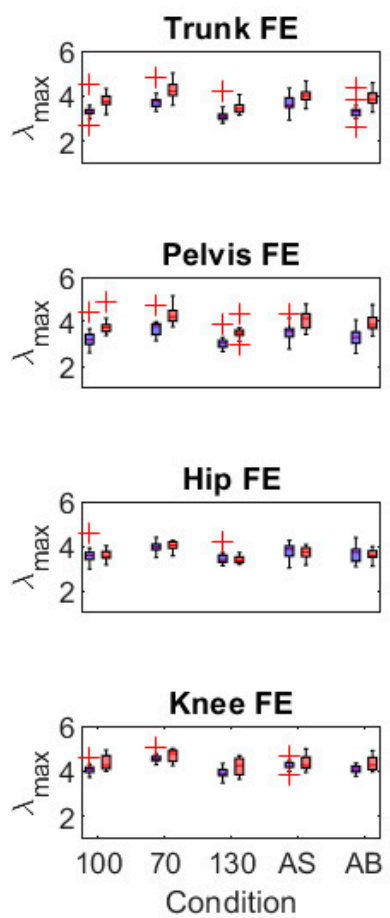

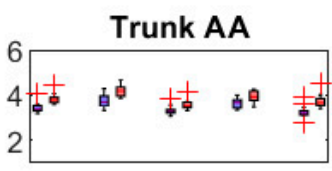

Pelvis AA

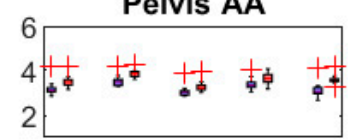

Hip AA

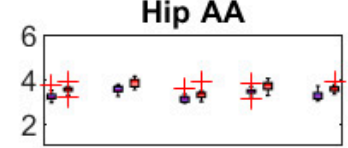

Ankle FE

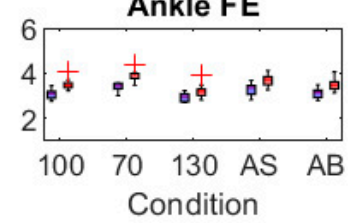

Trunk IE

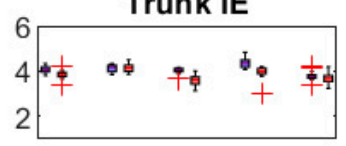

Pelvis IE

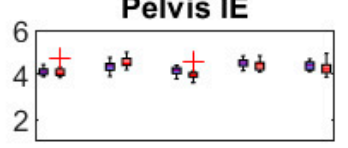

Hip IE

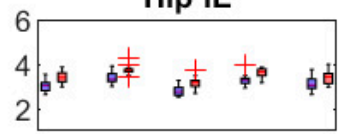

Ankle AA

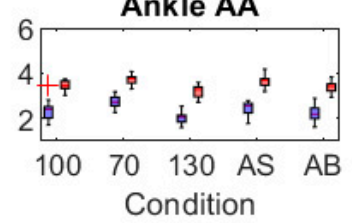

Figure 5. Boxplots of local divergence exponent $\left(\lambda_{\max }\right)$ of trunk, pelvis, and lower-limb joint angles for the optoelectronicdriven (blue) and IMU-driven (red) biomechanical models of constant-speed treadmill gait. Conditions displayed on the horizontal axis are preferred speed and arm swing (100), 70\% preferred speed (70), 130\% preferred speed (130), active arm swing ( $\mathrm{AS}$ ), and arms bound ( $\mathrm{AB})$. Red crosses are outliers $>2 *$ interquartile range. Angles include flexion/extension (FE), abduction/adduction (AA), and internal/external rotation (IE). 


\section{Discussion}

\subsection{Validity of IMU-Modelled Joint Angle Time Series}

Using a full-body biomechanical model with muscle-actuated lower limbs [33] to analyze several 7-min conditions of gait kinematics, our findings confirm that driving this model with IMUs (using the OpenSense open-source toolkit for OpenSim) produces, on average, accurate joint angle time series relative to the optoelectronic-driven model, with the exception of pelvis tilt, swing-phase hip rotation and swing-phase ankle inversion. RMSD across all gait speed and arm swing conditions averaged $5.3^{\circ}$; when ankle inversion was excluded from this calculation, RMSD averaged $4.8^{\circ}$, an accuracy viewed as acceptable for many clinical applications [29]. This amount of accuracy and the range in RMSD among individual joints $\left(1.7-7.5^{\circ}\right)$ agrees with recent findings from the developers $[14,15]$. One observation from our ensemble-averaged lower-limb joint angles (Figure 2) was that optoelectronic-IMU differences were notable in the non-sagittal plane during swing (e.g., hip abduction, hip rotation, and ankle inversion), potentially due to the relatively small ROMs of these angles in combination with the higher segmental velocities during swing. Nonetheless, we report an accuracy for hip rotation (pooled RMSD of $6.2^{\circ}$ ) that improved upon the developers' findings $\left(10-12^{\circ}\right)$. Because allowing ankle inversion/eversion in addition to dorsiflexion/plantarflexion did not affect the inverse kinematics solution for hip rotation (see, for example, Figure S1), we believe our improvement in accuracy is attributable to omitting magnetometer data when using Madgwick's gradient-descent fusion algorithm [21] and, instead, identifying negative $(<-0.0010 \mathrm{rad} / \mathrm{s})$ and positive $(>0.0010 \mathrm{rad} / \mathrm{s})$ linear orientation drifts and detrending these offline. Extending previous findings, we also found RMSDs of IMU-modelled trunk and pelvis angles $\left(1.7-10.6^{\circ}\right)$ that were similar to those of the joints in the lower limb. Thus, with the notable exceptions of pelvis tilt, hip rotation, and ankle inversion, our findings indicate that joint angle time series for the trunk and below can be estimated on average with acceptable accuracy during walking over a wide range of walking speeds and arm swing amplitudes.

\subsection{Validity of IMU-Modelled Joint Angle Outcomes}

The concurrent validity of the joint angle time series, however, did not extend to the discrete outcomes to the same extent. For ROM, as reported by Beange et al. [52,53], optoelectronic-IMU consistency was excellent in the primary plane of movement (trunk rotation, pelvis rotation, hip flexion, ankle dorsiflexion) with RMSDs similar to the corresponding time series (ROM: $2.0-7.7^{\circ}$; time series: $1.9-6.9^{\circ}$ ), but consistency was often poor in the non-primary movement planes. Therefore, measurement consistency of ROM for the secondary and tertiary planes was not improved by using a biomechanically constrained kinematic model. RMSDs for the secondary and tertiary planes $\left(1.8-8.5^{\circ}\right)$ were comparable to those of the primary movement plane, confirming the findings of Beange et al. $[52,53]$ and likely due to the lower ROMs about the secondary and tertiary planes; future IMUmodelling work of lower-limb joint kinematics could investigate whether consistency is higher in locomotor tasks with similar segmental velocities that include larger ROMs in the frontal and transverse planes, such as walking with side-stepping and/or turning. ROM was also below proposed limits of agreement of $\pm 10^{\circ}$ [54] for only 6 of the 12 degrees of freedom we analyzed, with underestimation biases (trunk rotation and ankle flexion) partly responsible. Together, these findings show that acceptable validity of a joint angle time series does not necessarily translate to acceptable validity in the discrete outcomes of that time series. Specifically, our IMU model estimates joint angle ROM accurately, but has inconsistencies in the non-primary movement planes and limitations in absolute agreement.

For motor variability outcomes, consistency was best for $\lambda_{\max }$ and meanSD, reaching good-excellent levels for nearly all degrees of freedom with RMSDs of $0.22-0.61^{\circ}$ and $0.14-0.61^{\circ}$, respectively. Our findings agree with the high optoelectronic-IMU consistency in $\lambda_{\max }$ reported for repetitive spine flexion/extension [55] and support the concurrent validity of IMU-based measurements of joint local dynamic stability. For the first time, we demonstrate high consistency and accuracy for joint angles with low range of motion and 
beyond the primary plane of movement, providing a new validated model for investigating magnitude of variability and local dynamic stability in tridimensional joint motion from stride to stride. Caution is advised regarding the absolute agreement of our outcomes since we were unable to identify limits of agreement in the literature for comparison and since the IMU-based model biases showed that $\lambda_{\max }$ and meanSD were each underestimated for trunk rotation but overestimated for hip, knee, and ankle angles.

DFA $\alpha$ and SaEn were not sufficiently consistent to be considered valid in their present computational form (DFA $\alpha \mathrm{ICC}_{2,1}:-0.05-0.65$; SaEn ICC $\left.2,1:-0.12-0.74\right)$. DFA $\alpha$ inconsistencies may be partly derived from $\mathrm{ROM}$ inconsistencies since we investigated fluctuations of this discrete metric. This is supported by our finding that the only two angles with good consistency in DFA $\alpha$ (trunk rotation, ankle dorsiflexion) also had excellent consistency in ROM, indicating that improvement in calculation of ROM is likely to produce more valid analyses of fluctuation persistence. SaEn inconsistencies may be due to changes in the information of joint angles in the IMU-driven model. The calculation of this metric for continuous series of joint angles is sensitive to the sampling frequency, the tolerance ratio $r$, and the embedding dimension $\mathrm{m}$ [48]. We resampled IMU-based joint angles to compensate for sampling frequency and selected $\mathrm{r}$ and $\mathrm{m}$ values which produced stable measurements in slow and fast gait [47]. However, specific tuning of SaEn parameters to IMU-based kinematic models is likely needed. Alternatively, calculation of SaEn may be more robust to tuning parameters by analyzing the discrete ROM series rather than the continuous joint angle series [48], but this also relies on improving the estimation of ROM. Therefore, use of IMU-based biomechanical models to investigate the persistence and regularity of joint angle fluctuations in gait requires improvements to the estimation of ROM and the identification of appropriate SaEn calculation parameters for IMU-derived joint angles.

\subsection{Sensitivity of IMU-Modelled Joint Angle Outcomes to Within-Participant Effects}

Our results show, for the first time, that joint angle outcomes estimated from an IMU-driven biomechanical model were also sensitive to within-participant responses detected by an optoelectronic-driven model in the majority of cases. Relative to gait at preferred speed and with preferred arm swing, responses to changes in speed and arm swing amplitude were detected identically between models for 36/48 comparisons for ROM, 27/48 comparisons for meanSD, and 34/48 comparisons for $\lambda_{\max }$. Model-detected responses are in agreement with several previous findings, including increases in joint ROM with fast speed and decreases with slow speed [56], increases in SD of trunk kinematics and meanSD of lower-limb angles with active arm swing [38,39], and increases in $\lambda_{\max }$ of hip abduction with active arm swing [39]. Although our trunk motion responses appear to disagree with reported increases in meanSD with fast speed [35], increases in $\lambda_{\max }$ with fast speed [36], and decreases in $\lambda_{\max }$ with active arm swing [37], differences can be attributed to the reference frame of the trunk (relative to the pelvis in our study vs. relative to ground in [35]) and to the sensitivity of the $\lambda_{\max }$ state-space to different inputs [57] (time-delayed joint angles in our study vs. velocities and accelerations [36] vs. timedelayed velocities [37]). In the minority of cases where our optoelectronic and IMU models detected different responses, directionality never disagreed, and differences were mostly due to the IMU-based model detecting no response when a change was detected by the optoelectronic-based model. In these cases, there were no statistically significant changes for the IMU-based model, but all means moved in the same direction as the optoelectronicbased model (Tables S1 and S2). As the probability of this due to chance is very low, it seems that IMU-modelled responses had smaller effect sizes than this study was powered to detect, due to smaller changes in magnitude and/or larger group variance. Furthermore, the excess limits of agreement for some ROMs did not influence sensitivity of the IMU model, as responses in ROM for angles exceeding limits of $\pm 10^{\circ}$ [54] were the same in many cases during gait at fast speed (trunk abduction, trunk rotation, ankle dorsiflexion), at slow speed (trunk abduction, trunk rotation, hip abduction, ankle dorsiflexion), with active 
arm swing (trunk abduction, pelvis flexion, hip abduction, knee flexion, ankle dorsiflexion, ankle inversion), and with bound arms (trunk rotation, ankle flexion, ankle inversion). Together, these findings show that the IMU-driven biomechanical model is sensitive to within-group responses in ROM, meanSD, and $\lambda_{\text {max }}$, and suggest that larger sample sizes may further improve sensitivity by compensating for smaller effect sizes.

\subsection{Limitations}

IMU-driven biomechanical simulations of movement are a new area for investigation and have important limitations. In our study, these were specific to the demographics of our sample, the biomechanical model, and how gait was studied. As a first step for modelling motor variability in gait, we recruited a convenience sample of healthy young adult males and females whose average BMI was in the "normal" range. Our motivation for investigating motor variability in gait, however, is to investigate stride-to-stride control of older adults as it relates to fall risk [3]. Confirmation of validity and sensitivity of an IMU-driven biomechanical model is still needed in older populations living with and without neurological conditions.

To mitigate differences attributed to different initial model poses in our evaluation of validity, we decided to offset the optoelectronic model to match the standing pose of the IMU model at the first frame, similar to the approach of Al Borno et al. [15]. This is unrealistic for situations where an optoelectronic system is unavailable to establish the initial IMU model pose. Adding the average correction (3.8 $[15])$ to the RMSD differences would produce differences that exceed limits tolerable for clinical applications [29]. However, ROM and motor variability were unaffected by this offset, and further IMU calibration procedures [58] are unlikely to affect validity or sensitivity of these measures. Thus, we expect that our conclusions on the validity of our outcomes can be generalized to situations where only IMU sensors are available.

Finally, we investigated performance of the IMU-driven model during treadmill gait, where stride-to-stride variability is lower than in overground gait $[59,60]$. Treadmills allow for analyses at constant speed and over a large number of continuous strides; for example, more than 100 strides are needed to reliably quantify SD of stride time and $\lambda_{\max }$ of tridimensional trunk acceleration [40]. Large numbers of strides can be recorded in optoelectronic-based overground studies when gait involves turning and strides are discontinuous [61] but calculation of local dynamic stability requires continuous time series. Use of a treadmill was the only practical way for us to quantify motor variability of straightline continuous walking with the metrics we calculated and using the optoelectronic and IMU motion capture systems simultaneously. Simultaneous measurement with these systems was necessary to test the validity of our model. We are unaware of a clear reason that our findings would not hold for overground gait since our IMU orientations do not rely on magnetometers, which are sensitive to fluctuations in heterogeneity of the local magnetic field, and the suitability of IMU-driven biomechanical models for joint kinematic evaluations in overground locomotion is promising $[14,15,27]$. Nonetheless, the sensitivity of the IMU-driven model should be confirmed for overground evaluations of stride-to-stride control.

\section{Conclusions}

In summary, excluding pelvis tilt and swing-phase hip rotation and ankle inversion, IMU-based joint angle time series were acceptably accurate from the trunk down, ROM was acceptably consistent and accurate in the primary plane of motion, and magnitude of variability and local dynamic stability were acceptably consistent and accurate in all planes of motion. Validity was supported by the sensitivity of the IMU model to gait speed and arm swing amplitude-related responses in ROM, magnitude of variability, and local dynamic stability in the majority of cases. However, IMU-modelled estimates of ROM fluctuation persistence and of angle regularity were not acceptably consistent or accurate. We conclude that, for moderate-duration walking at slow and fast speeds, the 
IMU-driven, magnetometer-free, open-source biomechanical model used in this study provides valid estimates of joint angle time series, ROM in the primary plane of motion, stride-to-stride magnitude of variability, and stride-to-stride local dynamic stability which are also sensitive to within-participant responses. This provides a new way to evaluate biomechanical control of walking outside of the lab and clinic.

Supplementary Materials: The following are available online at https:/ /www.mdpi.com/article/10 $.3390 / s 21227690 / s 1$, Figure S1: Inverse kinematics solutions to optoelectronic-based models with and without upper-limb weights, and to optoelectronic- and IMU-based models for 1- and 2-degreeof-freedom ankles, Figure S2: Removal of orientation drift from IMUs, Figure S3: Identification of heel strikes from optoelectronic- and IMU-modelled calcaneus linear velocities, Figure S4: Rootmean-squared difference of IMU-based joint angles relative to optoelectronic motion capture as a function of stride number, Figures S5-S9: Bland-Altman plots of joint angle range of motion and joint angle variability outcomes, Tables S1 and S2: Summarized optoelectronic- and IMU-based joint angle outcome data and sensitivity to gait speed and arm swing differences, Videos S1-S4: Animations of the optoelectronic-based and IMU-based biomechanical models for preferred speed and arm swing gait in a representative subject.

Author Contributions: Conceptualization, C.A.B., T.K.U., J.N. and R.B.G.; data curation, C.A.B.; formal analysis, C.A.B., T.K.U., J.N. and R.B.G.; funding acquisition, C.A.B., J.N. and R.B.G.; investigation, C.A.B.; methodology, C.A.B., T.K.U., J.N. and R.B.G.; project administration, C.A.B., J.N. and R.B.G.; software, C.A.B.; supervision, J.N. and R.B.G.; validation, C.A.B., T.K.U., J.N. and R.B.G.; visualization, C.A.B.; writing—original draft, C.A.B.; writing—review and editing, C.A.B., T.K.U., J.N. and R.B.G. All authors have read and agreed to the published version of the manuscript.

Funding: This research was funded by a postdoctoral fellowship from the uOttawa-Children's Hospital of Eastern Ontario Research Institute awarded to C.A.B., by grants from the Natural Sciences and Engineering Research Council of Canada awarded to J.N. (RGPIN-2016-04928) and R.B.G. (RGPIN-2020-04748), and by Ontario Early Researcher Awards to J.N. (ER16-12-206) and R.B.G. (ER17-13-007).

Institutional Review Board Statement: This study was conducted according to the guidelines of the Declaration of Helsinki, and approved by the institutional Research Ethics Board of the University of Ottawa (H-01-21-6261).

Informed Consent Statement: Informed consent was obtained from all participants involved in this study.

Data Availability Statement: The models underlying the findings of this study are freely available on SimTK (https://simtk.org/projects/bailey2021_imu (accessed on 2 November 2021)).

Acknowledgments: The authors thank Alexandre Mir-Orefice and Isabel Coll for their assistance in acquiring and processing some of the data from this study.

Conflicts of Interest: The authors declare no conflict of interest.

\section{References}

1. Newell, K.M.; Slifkin, A.B. The Nature of Movement Variability. In Motor Behavior and Human Skill: A Multidisciplinary Perspective; Human Kinetics: Champaign, IL, USA, 1998; pp. 143-160.

2. Hausdorff, J.M.; Rios, D.A.; Edelberg, H.K. Gait variability and fall risk in community-living older adults: A 1-year prospective study. Arch. Phys. Med. Rehabil. 2001, 82, 1050-1056. [CrossRef] [PubMed]

3. Hausdorff, J.M. Gait variability: Methods, modeling and meaning. J. Neuroeng. Rehabil. 2005, 2, 19. [CrossRef] [PubMed]

4. Buzzi, U.H.; Stergiou, N.; Kurz, M.; Hageman, P.; Heidel, J. Nonlinear dynamics indicates aging affects variability during gait. Clin. Biomech. 2003, 18, 435-443. [CrossRef]

5. Kurz, M.J.; Stergiou, N. The aging humans neuromuscular system expresses less certainty for selecting joint kinematics during gait. Neurosci. Lett. 2003, 348, 155-158. [CrossRef]

6. Bailey, C.A.; Porta, M.; Pilloni, G.; Arippa, F.; Côté, J.N.; Pau, M. Does variability in motor output at individual joints predict stride time variability in gait? Influences of age, sex, and plane of motion. J. Biomech. 2019, 99, 109574. [CrossRef]

7. Ihlen, E.A.F.; van Schooten, K.S.; Bruijn, S.M.; van Dieën, J.H.; Vereijken, B.; Helbostad, J.L.; Pijnappels, M. Improved Prediction of Falls in Community-Dwelling Older Adults Through Phase-Dependent Entropy of Daily-Life Walking. Front. Aging Neurosci. 2018, 10, 44. [CrossRef] 
8. Picerno, P. 25 years of lower limb joint kinematics by using inertial and magnetic sensors: A review of methodological approaches. Gait Posture 2017, 51, 239-246. [CrossRef]

9. Zhang, J.-T.; Novak, A.; Brouwer, B.; Li, Q. Concurrent validation of Xsens MVN measurement of lower limb joint angular kinematics. Physiol. Meas. 2013, 34, N63-N69. [CrossRef]

10. Palermo, E.; Rossi, S.; Marini, F.; Patane, F.; Cappa, P. Experimental evaluation of accuracy and repeatability of a novel body-tosensor calibration procedure for inertial sensor-based gait analysis. Measurement 2014, 52, 145-155. [CrossRef]

11. Zihajehzadeh, S.; Park, E.J. A Novel Biomechanical Model-Aided IMU/UWB Fusion for Magnetometer-Free Lower Body Motion Capture. IEEE Trans. Syst. Man Cybern. Syst. 2016, 47, 927-938. [CrossRef]

12. Teufl, W.; Miezal, M.; Taetz, B.; Fröhlich, M.; Bleser, G. Validity of inertial sensor based 3D joint kinematics of static and dynamic sport and physiotherapy specific movements. PLoS ONE 2019, 14, e0213064. [CrossRef] [PubMed]

13. Rapp, E.; Shin, S.; Thomsen, W.; Ferber, R.; Halilaj, E. Estimation of kinematics from inertial measurement units using a combined deep learning and optimization framework. J. Biomech. 2021, 116, 110229. [CrossRef] [PubMed]

14. Slade, P.; Habib, A.; Hicks, J.L.; Delp, S.L. An open-source and wearable system for measuring 3D human motion in real-time. IEEE Trans. Biomed. Eng. 2021, 1. [CrossRef] [PubMed]

15. Al Borno, M.; O’Day, J.; Ibarra, V.; Dunne, J.; Seth, A.; Habib, A.; Ong, C.; Hicks, J.; Uhlrich, S.; Delp, S. OpenSense: An open-source tool box for Inertial-Measurement-Unit-based measurement of lower extremity kinematics over long durations. bioRxiv 2021, 1-26. [CrossRef]

16. Ferrari, A.; Cutti, A.G.; Garofalo, P.; Raggi, M.; Heijboer, M.; Cappello, A.; Davalli, A. First in vivo assessment of "Outwalk”: A novel protocol for clinical gait analysis based on inertial and magnetic sensors. Med Biol. Eng. Comput. 2009, 48, 1-15. [CrossRef] [PubMed]

17. Al-Amri, M.; Nicholas, K.; Button, K.; Sparkes, V.; Sheeran, L.; Davies, J.L. Inertial Measurement Units for Clinical Movement Analysis: Reliability and Concurrent Validity. Sensors 2018, 18, 719. [CrossRef] [PubMed]

18. De Villa, S.G.; Munoz Diaz, E.; Ahmed, D.B.; Jimenez Martin, A.; Dominguez, J.J.G. IMU-based Characterization of the Leg for the Implementation of Biomechanical Models. In Proceedings of the 2019 International Conference on Indoor Positioning and Indoor Navigation (IPIN), Pisa, Italy, 30 September-3 October 2019; Volume 17, pp. 1-8.

19. Weygers, I.; Kok, M.; Konings, M.; Hallez, H.; De Vroey, H.; Claeys, K. Inertial Sensor-Based Lower Limb Joint Kinematics: A Methodological Systematic Review. Sensors 2020, 20, 673. [CrossRef]

20. Roetenberg, D.; Luinge, H.; Slycke, P. Xsens MVN: Full 6DOF Human Motion Tracking Using Miniature Inertial Sensors. Xsens Motion Technologies BV, Tech. Rep 1. 2009, pp. 1-7. Available online: http:/ / citeseerx.ist.psu.edu/viewdoc/download?doi=10.1 $.1 .569 .9604 \&$ rep $=$ rep1\&type $=$ pdf (accessed on 21 June 2021).

21. Madgwick, S.O.H.; Harrison, A.J.L.; Vaidyanathan, R. Estimation of IMU and MARG orientation using a gradient descent algorithm. In Proceedings of the 2011 IEEE International Conference on Rehabilitation Robotics, Zurich, Switzerland, 29 June-1 July 2011; pp. 1-7.

22. Šlajpah, S.; Kamnik, R.; Munih, M. Kinematics based sensory fusion for wearable motion assessment in human walking. Comput. Methods Programs Biomed. 2014, 116, 131-144. [CrossRef]

23. O’Donovan, K.J.; Kamnik, R.; O'Keeffe, D.T.; Lyons, G.M. An inertial and magnetic sensor based technique for joint angle measurement. J. Biomech. 2007, 40, 2604-2611. [CrossRef] [PubMed]

24. Ibata, Y.; Kitamura, S.; Motoi, K.; Sagawa, K. Measurement of three-dimensional posture and trajectory of lower body during standing long jumping utilizing body-mounted sensors. In Proceedings of the 2013 35th Annual International Conference of the IEEE Engineering in Medicine and Biology Society (EMBC), Osaka, Japan, 3-7 July 2013; pp. 4891-4894.

25. De Vries, W.; Veeger, D.; Baten, C.; van der Helm, F. Magnetic distortion in motion labs, implications for validating inertial magnetic sensors. Gait Posture 2009, 29, 535-541. [CrossRef]

26. Meng, X.; Zhang, Z.; Wu, J.-K.; Wong, L. Hierarchical Information Fusion for Global Displacement Estimation in Microsensor Motion Capture. IEEE Trans. Biomed. Eng. 2013, 60, 2052-2063. [CrossRef]

27. Kok, M.; Hol, J.D.; Schön, T. An optimization-based approach to human body motion capture using inertial sensors. IFAC Proc. Vol. 2014, 47, 79-85. [CrossRef]

28. Tagliapietra, L.; Modenese, L.; Ceseracciu, E.; Mazzà, C.; Reggiani, M. Validation of a model-based inverse kinematics approach based on wearable inertial sensors. Comput. Methods Biomech. Biomed. Eng. 2018, 21, 834-844. [CrossRef] [PubMed]

29. McGinley, J.L.; Baker, R.; Wolfe, R.; Morris, M.E. The reliability of three-dimensional kinematic gait measurements: A systematic review. Gait Posture 2009, 29, 360-369. [CrossRef]

30. Delp, S.L.; Anderson, F.C.; Arnold, A.S.; Loan, P.; Habib, A.; John, C.T.; Guendelman, E.; Thelen, D.G. OpenSim: Open-Source Software to Create and Analyze Dynamic Simulations of Movement. IEEE Trans. Biomed. Eng. 2007, 54, 1940-1950. [CrossRef] [PubMed]

31. Seth, A.; Hicks, J.L.; Uchida, T.K.; Habib, A.; Dembia, C.L.; Dunne, J.J.; Ong, C.; Demers, M.S.; Rajagopal, A.; Millard, M.; et al. OpenSim: Simulating musculoskeletal dynamics and neuromuscular control to study human and animal movement. PLoS Comput. Biol. 2018, 14, e1006223. [CrossRef]

32. Faul, F.; Erdfelder, E.; Lang, A.-G.; Buchner, A. G*Power 3: A flexible statistical power analysis program for the social, behavioral, and biomedical sciences. Behav. Res. Methods 2007, 39, 175-191. [CrossRef] [PubMed] 
33. Rajagopal, A.; Dembia, C.; DeMers, M.; Delp, D.D.; Hicks, J.L.; Delp, S.L. Full-Body Musculoskeletal Model for Muscle-Driven Simulation of Human Gait. IEEE Trans. Biomed. Eng. 2016, 63, 2068-2079. [CrossRef]

34. Dingwell, J.B.; Marin, L.C. Kinematic variability and local dynamic stability of upper body motions when walking at different speeds. J. Biomech. 2006, 39, 444-452. [CrossRef]

35. Kang, H.G.; Dingwell, J. Separating the effects of age and walking speed on gait variability. Gait Posture 2008, $27,572-577$. [CrossRef]

36. Kang, H.G.; Dingwell, J. Effects of walking speed, strength and range of motion on gait stability in healthy older adults. J. Biomech. 2008, 41, 2899-2905. [CrossRef] [PubMed]

37. Hill, A.; Nantel, J. The effects of arm swing amplitude and lower-limb asymmetry on gait stability. PLoS ONE 2019, 14, e0218644. [CrossRef]

38. Siragy, T.; Mezher, C.; Hill, A.; Nantel, J. Active arm swing and asymmetric walking leads to increased variability in trunk kinematics in young adults. J. Biomech. 2019, 99, 109529. [CrossRef]

39. Bailey, C.A.; Hill, A.; Graham, R.B.; Nantel, J. Effects of arm swing amplitude and lower limb asymmetry on motor variability patterns during treadmill gait. J. Biomech. 2021, 130, 110855. [CrossRef]

40. Riva, F.; Bisi, M.C.; Stagni, R. Gait variability and stability measures: Minimum number of strides and within-session reliability. Comput. Biol. Med. 2014, 50, 9-13. [CrossRef]

41. Enright, P.L. The six-minute walk test. Respir. Care 2003, 48, 783-785. [PubMed]

42. Woltring, H.J. A Fortran package for generalized, cross-validatory spline smoothing and differentiation. Adv. Eng. Softw. (1978) 1986, 8, 104-113. [CrossRef]

43. Wu, Y.; Li, Y.; Liu, A.-M.; Xiao, F.; Wang, Y.-Z.; Hu, F.; Chen, J.-L.; Dai, K.-R.; Gu, D.-Y. Effect of active arm swing to local dynamic stability during walking. Hum. Mov. Sci. 2016, 45, 102-109. [CrossRef] [PubMed]

44. Peng, C.-K.; Buldyrev, S.; Goldberger, A.L.; Havlin, S.; Sciortino, F.; Simons, M.; Stanley, H.E. Long-range correlations in nucleotide sequences. Nature 1992, 356, 168-170. [CrossRef] [PubMed]

45. Hausdorff, J.M.; Peng, C.-K.; Ladin, Z.; Wei, J.Y.; Goldberger, A.L. Is walking a random walk? Evidence for long-range correlations in stride interval of human gait. J. Appl. Physiol. 1995, 78, 349-358. [CrossRef]

46. Dingwell, J.B.; Cusumano, J.P. Identifying Stride-To-Stride Control Strategies in Human Treadmill Walking. PLoS ONE 2015, 10, e0124879. [CrossRef]

47. Costa, M.; Peng, C.-K.; Goldberger, A.L.; Hausdorff, J.M. Multiscale entropy analysis of human gait dynamics. Phys. A Stat. Mech Its Appl. 2003, 330, 53-60. [CrossRef]

48. McCamley, J.D.; Denton, W.; Arnold, A.; Raffalt, P.C.; Yentes, J.M. On the Calculation of Sample Entropy Using Continuous and Discrete Human Gait Data. Entropy 2018, 20, 764. [CrossRef] [PubMed]

49. Cicchetti, D.V. Guidelines, criteria, and rules of thumb for evaluating normed and standardized assessment instruments in psychology. Psychol. Assess. 1994, 6, 284-290. [CrossRef]

50. Benjamini, Y.; Hochberg, Y. Controlling the False Discovery Rate: A Practical and Powerful Approach to Multiple Testing. J. R. Stat. Soc. Ser. B Methodol. 1995, 57, 289-300. [CrossRef]

51. Ko, S.-U.; Tolea, M.I.; Hausdorff, J.M.; Ferrucci, L. Sex-specific differences in gait patterns of healthy older adults: Results from the Baltimore Longitudinal Study of Aging. J. Biomech. 2011, 44, 1974-1979. [CrossRef]

52. Beange, K.H.E.; Chan, A.D.C.; Graham, R.B. Evaluation of wearable IMU performance for orientation estimation and motion tracking. In Proceedings of the IEEE International Workshop on Medical Measurement and Applications, Rome, Italy, 11-13 June 2018; pp. 1-6.

53. Beange, K.H.E.; Chan, A.D.C.; Graham, R.B. Wearable sensor performance for motion tracking of the lumbar spine. In Proceedings of the 42nd Canadian Medical and Biological Engineering Conference, Ottawa, ON, Canada, 21-24 May 2019; pp. 1-4.

54. El-Zayat, B.F.; Efe, T.; Heidrich, A.; Anetsmann, R.; Timmesfeld, N.; Fuchs-Winkelmann, S.; Schofer, M.D. Objective assessment, repeatability, and agreement of shoulder ROM with a 3D gyroscope. BMC Musculoskelet. Disord. 2013, 14, 72. [CrossRef]

55. Beange, K.H.; Chan, A.D.; Beaudette, S.M.; Graham, R.B. Concurrent validity of a wearable IMU for objective assessments of functional movement quality and control of the lumbar spine. J. Biomech. 2019, 97, 109356. [CrossRef] [PubMed]

56. Pietraszewski, B.; Winiarski, S.; Jaroszczuk, S. Three-dimensional human gait pattern-reference data for normal men. Acta Bioeng. Biomech. 2012, 14, 9-16. [CrossRef]

57. Gates, D.H.; Dingwell, J.B. Comparison of different state space definitions for local dynamic stability analyses. J. Biomech. 2009, 42, 1345-1349. [CrossRef]

58. Pacher, L.; Chatellier, C.; Vauzelle, R.; Fradet, L. Sensor-to-Segment Calibration Methodologies for Lower-Body Kinematic Analysis with Inertial Sensors: A Systematic Review. Sensors 2020, 20, 3322. [CrossRef] [PubMed]

59. Dingwell, J.; Cusumano, J.P.; Cavanagh, P.R.; Sternad, D. Local Dynamic Stability Versus Kinematic Variability of Continuous Overground and Treadmill Walking. J. Biomech. Eng. 2000, 123, 27-32. [CrossRef] [PubMed]

60. Hollman, J.H.; Watkins, M.K.; Imhoff, A.C.; Braun, C.E.; Akervik, K.A.; Ness, D.K. A comparison of variability in spatiotemporal gait parameters between treadmill and overground walking conditions. Gait Posture 2016, 43, 204-209. [CrossRef]

61. König, N.; Singh, N.; von Beckerath, J.; Janke, L.; Taylor, W. Is gait variability reliable? An assessment of spatio-temporal parameters of gait variability during continuous overground walking. Gait Posture 2014, 39, 615-617. [CrossRef] [PubMed] 